\title{
Dual epithelial and immune cell function of Dvl1 regulates gut microbiota composition and intestinal homeostasis
}

\author{
Haim Belinson, ${ }^{1,2}$ Adam K. Savage, ${ }^{3}$ Douglas Fadrosh, ${ }^{4}$ Yien-Ming Kuo, ${ }^{4}$ Din Lin,${ }^{4}$ \\ Ricardo Valladares, ${ }^{4}$ Ysbrand Nusse, ${ }^{1,2}$ Anthony Wynshaw-Boris, ${ }^{1,5}$ Susan V. Lynch, ${ }^{4}$ \\ Richard M. Locksley, ${ }^{3,4}$ and Ophir D. Klein ${ }^{1,2}$ \\ 'Department of Pediatrics and Institute for Human Genetics, ${ }^{2}$ Department of Orofacial Sciences and Program in \\ Craniofacial Biology, ${ }^{3}$ Howard Hughes Medical Institute, Department of Microbiology \& Immunology, and ${ }^{4}$ Department of \\ Medicine, University of California, San Francisco, California, USA. ${ }^{5}$ Department of Genetics and Genome Sciences, \\ Case Western Reserve University School of Medicine, Cleveland, Ohio, USA.
}

\begin{abstract}
Homeostasis of the gastrointestinal (CI) tract is controlled by complex interactions between epithelial and immune cells and the resident microbiota. Here, we studied the role of Wnt signaling in $\mathrm{GI}$ homeostasis using Disheveled 1 knockout (Dv/1/-) mice, which display an increase in whole gut transit time. This phenotype is associated with a reduction and mislocalization of Paneth cells and an increase in CD8 ${ }^{+} \mathrm{T}$ cells in the lamina propria. Bone marrow chimera experiments demonstrated that $\mathrm{GI}$ dysfunction requires abnormalities in both epithelial and immune cells. Dv/1-1- mice exhibit a significantly distinct GI microbiota, and manipulation of the gut microbiota in mutant mice rescued the $\mathrm{Gl}$ transit abnormality without correcting the Paneth and $\mathrm{CD8}^{+} \mathrm{T}$ cell abnormalities. Moreover, manipulation of the gut microbiota in wild-type mice induced a GI transit abnormality akin to that seen in $\mathrm{Dvl1}^{-I^{-}}$mice. Together, these data indicate that microbiota manipulation can overcome host dysfunction to correct GI transit abnormalities. Our findings illustrate a mechanism by which the epithelium and immune system coregulate gut microbiota composition to promote normal GI function.
\end{abstract}

Conflict of interest: The authors have declared that no conflict of interest exists.

Submitted: November 5, 2015

Accepted: June 2, 2016

Published: July 7, 2016

Reference information: JCI Insight. 2016;1(10):e85395. doi:10.1172/jici.insight.85395.

\section{Introduction}

The intestinal epithelium is comprised of a diversity of cell types that are responsible for the various functions of the organ, including absorption of nutrients and secretion of protective mucus, antimicrobial peptides, and hormones. The specialized cell types involved in these functions include Paneth cells, enterocytes, goblet cells, enteroendocrine cells (1), and tuft cells (2).

Homeostasis of the gastrointestinal (GI) tract is regulated by interactions between constituents of the intestinal ecosystem, including epithelial and immune cell populations and the resident microbiota. The murine intestine represents a complex host-microbial ecosystem housing hundreds of distinct bacterial species that engage in a bidirectional exchange of signals. Alterations in gut microbiota composition (dysbiosis) may cause immune dysregulation, resulting in pathogenic effects on GI function (3). Paneth cells, which are part of the epithelial crypts, play a pivotal role in controlling host-commensal balance (4) via the secretion of a diverse repertoire of antimicrobial proteins (5). In addition, production of several antimicrobial peptides is regulated by bacterial signals (5-7). Importantly, Paneth cells are essential for controlling intestinal barrier penetration by commensal and pathogenic bacteria (8).

In homeostasis, the intestinal immune cells maintain a balance between defense against pathogens and tolerance towards luminal antigens $(9,10)$. Mucosal innate immune responses are essential for eliciting adaptive immune responses, which may become the major drivers of chronic tissue inflammation (11). It is known that cytotoxic $\mathrm{CD}^{+} \mathrm{T}$ cells can escape both central and peripheral tolerance and may trigger autoimmune reactions to a microbial mimic of self-antigen (12). In addition, previous studies supported the role of cytotoxic $\mathrm{CD}^{+} \mathrm{T}$ cells in response to self- or exogenous antigens in the intestinal mucosa and suggested that the cytotoxic function of $\mathrm{CD}^{+} \mathrm{T}$ cells against epithelial cells may initiate the intestinal inflammatory process $(13,14)$. 
A

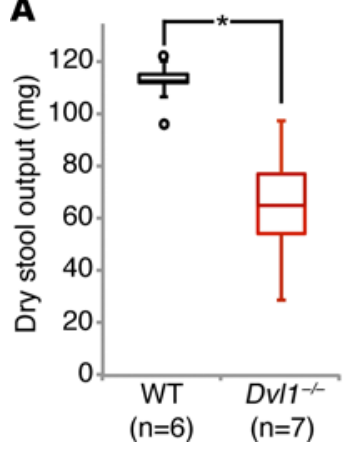

B

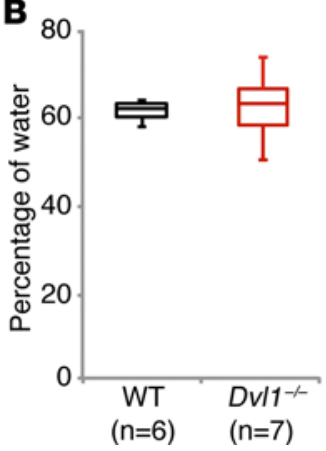

C

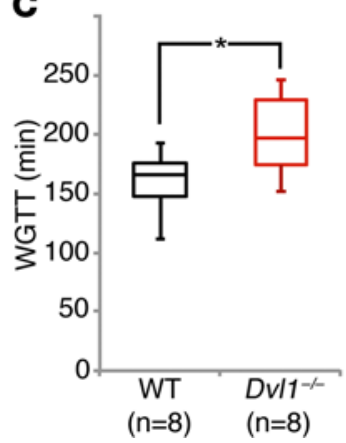

D

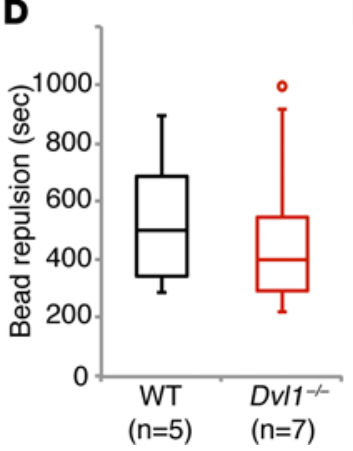

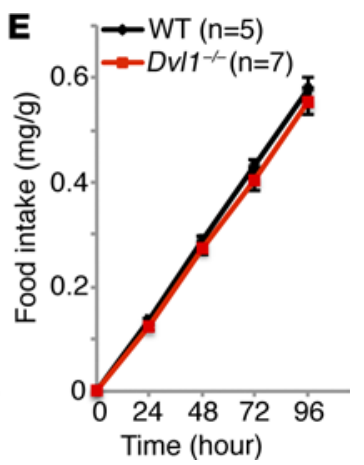

Figure 1. Abnormal GI function in Dv/1/-- mice. Twelve-week-old wild-type (WT) and Disheveled 1 knockout (Dv/1/-) mice were tested in a battery of gastrointestinal (GI) function tests. (A and B) Stool produced over a 1-hour time period was measured, and dry stool output (A) and percentage of water in the stool (B) were calculated. Results are presented as box plot, Bonferroni ${ }^{*} P<0.001$ for comparison of the $D v / 1^{-/-}$dry stool weight with those from WT mice. (C) Whole gut transit time (WCTT) was measured and results are presented as box plot, Bonferroni ${ }^{*} P<0.04$ for comparison of the Dv/1 ${ }^{-/-}$mice WGTT with those from WT mice. (D) Colonic motility was measured by the bead expulsion test and time to expel bead was recorded. Results are presented as box plot. (E) Whole-animal metabolic analysis was performed and food intake was measured. Results are presented as mean \pm SEM.

An important regulator of development and homeostasis in the GI tract is the Wnt signaling pathway $(15,16)$. Wnt signals are relayed from cell surface receptors to intracellular effectors by scaffold proteins of the Disheveled (Dvl) family. Wnt signals can be transduced through multiple pathways, including canonical Wnt/ $\beta$-catenin, planar cell polarity (PCP), and Wnt/ $\mathrm{Ca}^{2+}$ cascades. In the mammalian GI tract, the self-renewal capacity of epithelial stem cells is dependent on Wnt ligands provided by Paneth cells; the Paneth and stem cells normally remain in the crypts while the other cell types migrate up the villi (17-22). In the immune system, Wnt signaling controls the proliferation of $\mathrm{T}$ cell progenitors (23) and is thought to affect the cell-fate decisions of stem cells (24). Previous studies have indicated that Wnt signaling also regulates effector $\mathrm{T}$ cell development (25-27), regulatory $\mathrm{T}$ cell activation (28), and dendritic cell maturation (29), implicating Wnt signaling in the control of microbial populations in mammalian systems. Thus, Wnt signaling is important in a broad array of cell types. Dysregulation of Wnt signaling can therefore affect multiple cell types within an organ and, theoretically, could regulate the microbiome indirectly as well through effects on immune or other cells.

In this study, we asked whether and how Dvll regulates intestinal homeostasis. We used in vivo and in vitro approaches to examine the constituents of the intestinal ecosystem: epithelial and immune cells as well as the resident microbiota. We discovered that global deletion of Dvll causes dysfunction of Paneth cells in concert with an increase of $\mathrm{CD}^{+} \mathrm{T}$ cells in the GI tract, resulting in an abnormal microbial composition that elicited aberrant GI function.

\section{Results}

Abnormal GI function in Dvl1 ${ }^{-1-}$ mice. To study the role of Dvl-mediated Wnt signaling in homeostasis of the gut, we first asked if adult $D v l 1^{-/-}$mice, which are viable and fertile, have an intestinal phenotype; due to early lethality in $\sim 50 \%$ of $D v l 2$ and $100 \%$ of $D v l 3$ single null mutants, we opted not to examine those genotypes (30-32). To assess GI physiology in $D v l 1^{-1-}$ mice, we measured stool output over a 1-hour period. We found that dry stool output was reduced in $D v l 1^{-1-}$ mice compared to wild-type (WT) mice (Figure 1A), while the percentage of water in the stool was unchanged (Figure 1B), suggesting slower GI motility in the mutant mice. Using an oral gavage of carmine dye, we measured the whole gut transit time (WGTT) and found that this was longer in $D v l 1^{-/-}$mice compared to WT mice (Figure 1C), which explained the reduced stool output in $\mathrm{Dvl1^{-/- }}$ mice. Measurement of distal colonic motility, using the bead repulsion assay, revealed that $\mathrm{Dvl1^{-/- }}$ and WT mice were comparable (Figure 1D), indicating that the observed motility deficits originated from the small intestine and/or the proximal colon.

As the reduction in stool output could potentially result from a change in the metabolism of $D v l 1^{-1}$ mice, we housed control and mutant mice in metabolic cages over 5 days to measure gross physiological parameters, such as feeding, drinking, heat production, and activity. All parameters in the $D v l 1^{-1-}$ mice were similar to WT mice (Figure 1E and Supplemental Figure 1, A-E; supplemental material available online with this article; doi:10.1172/jci.insight.85395DS1), suggesting that the increased WGTT and reduced 


\section{A}

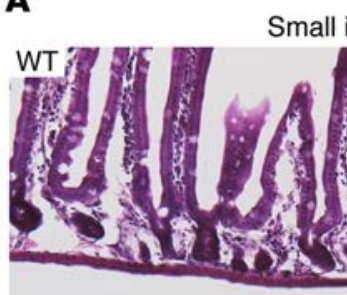

mall intestine
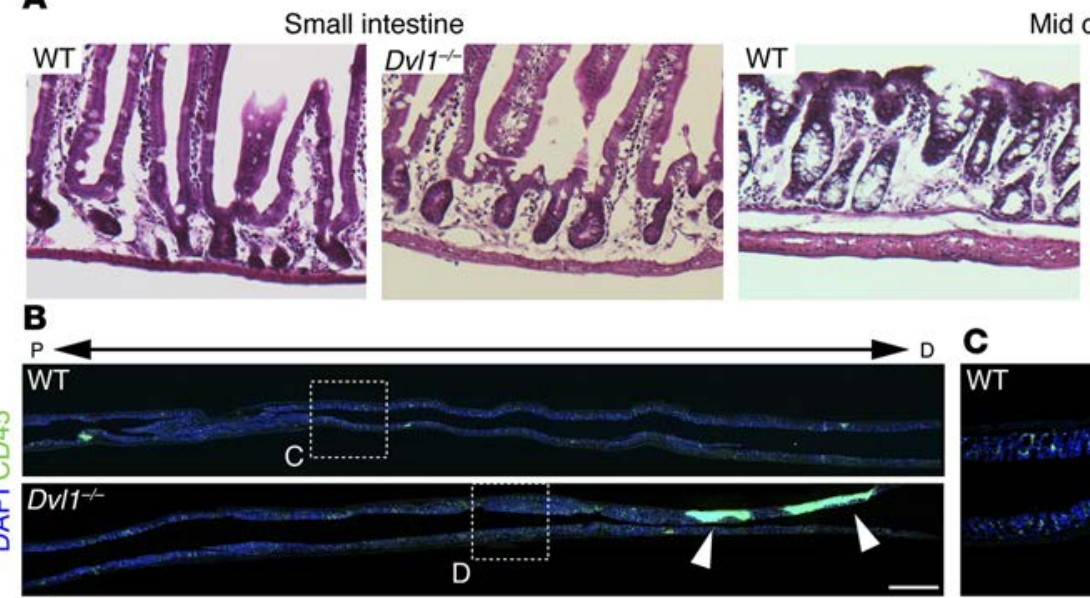

C
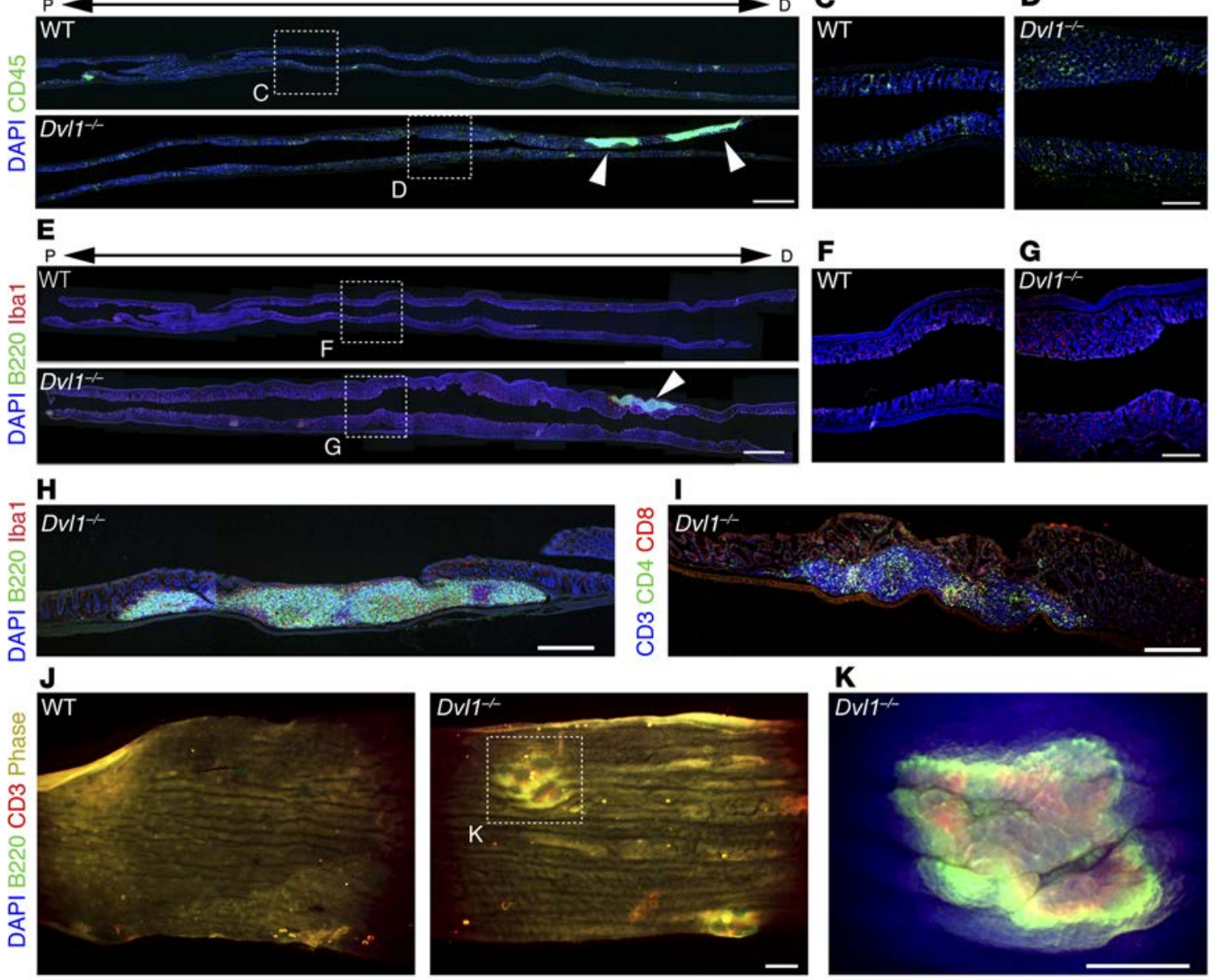

F

G
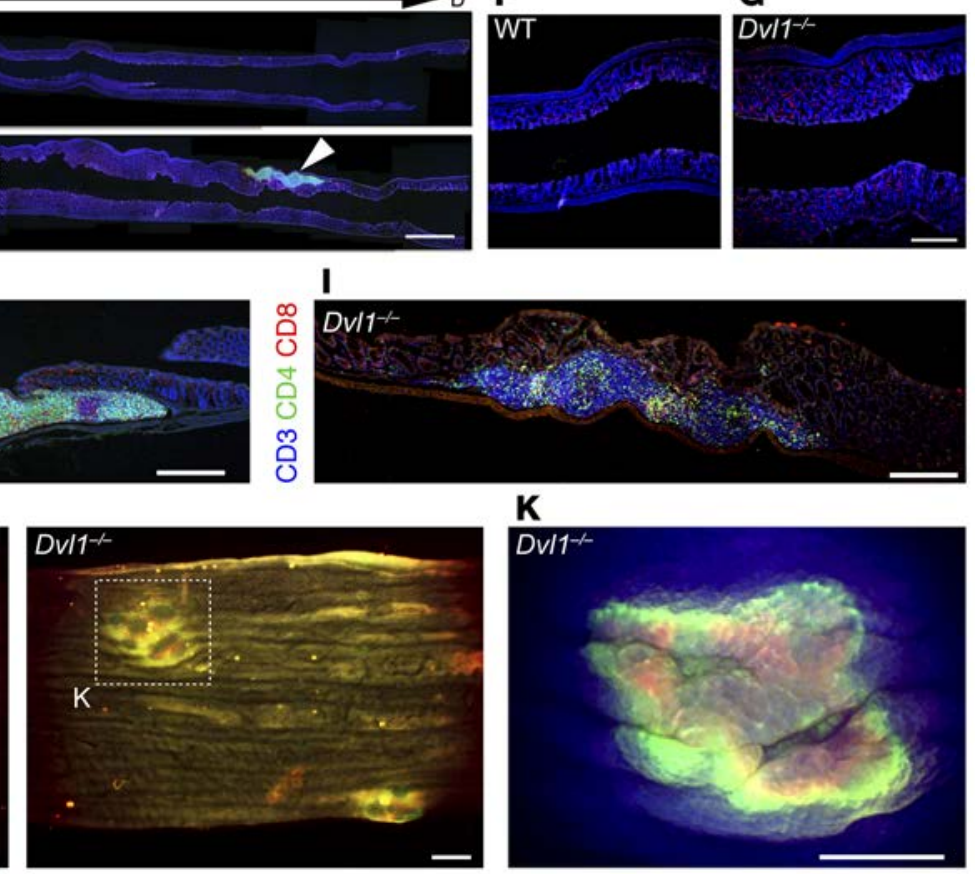

K

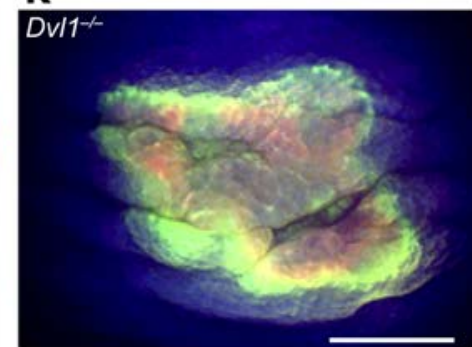

Figure 2. Abnormal colonic immune response in Dv/1/- mice. The gastrointestinal tracts of 12-week-old wild-type (WT) and Disheveled 1 knockout (Dv/1/-) mice were processed for histology. (A) Sections were stained with hematoxylin and eosin, and representative images are shown. Scale bar: $100 \mu \mathrm{m}$. (B) Sections of proximal colon were stained with DAPI (nuclei) and immunostained for CD45, and representative images are shown. Images are oriented from proximal (P) to distal (D), from left to right, respectively. Arrowheads denote the patch structures. Scale bar: 1,000 $\mu \mathrm{m}$. (C and D) Enlargement of inserts in B. Scale bar: $250 \mu \mathrm{m}$. (E) Sections of proximal colon were stained with DAPI and immunostained for B220 and Iba-1, and representative images are shown. Images are oriented from proximal $(P)$ to distal (D), from left to right, respectively. Arrowheads denote the patch structure. Scale bar: 1,000 $\mu \mathrm{m}$. (F and $\mathbf{C})$ Enlargement of inserts in E. Scale bar: $250 \mu \mathrm{m}$. (H) Sections from proximal colon of Dv/1/-- mice were stained with DAPI and immunostained for B220 and Iba-1, and a representative image of the patch region is shown. Scale bar: $100 \mu \mathrm{m}$. (I) Sections from proximal colon of Dv/1/-- mice were immunostained for CD3, CD4, and CD8, and a representative image of the patch region is shown. Scale bar: $100 \mu \mathrm{m}$. (J) Whole colons from WT and Dv/1 ${ }^{-1-}$ mice were fixed and stained with DAPI and immunostained for B220 and CD3, and representative images from the mid colon region are shown. Scale bar: $500 \mu \mathrm{m}$. (K) Enlargement of inserts in F. Scale bar: $2,000 \mu \mathrm{m}$.

1-hour dry stool output are not a result of altered gross metabolic changes in $D v l 1^{-/-}$mice but rather due to abnormal function within the GI tract itself.

Abnormal GI immune response in $D v 1^{-{ }^{-}}$mice. To begin to identify the cellular mechanisms underlying the abnormal GI function in Dvll mutants, we analyzed the GI tract using hematoxylin and eosin (H\&E) staining. We found that, whereas the small intestine was grossly normal in appearance, the proximal colon had patches of densely clustered cells in all $D v l 1^{-/-}$mice (6 of 6 ) but in none of the WT mice $(0$ of 4$)$ (Figure 2A). Immunostaining revealed that all cells in this patch were positive for CD45, a hematopoietic 
cell marker (Figure 2B). In addition, staining for B220 (B cells), Iba-1 (macrophages, Figure 2, E-H), and CD4 and CD8 (T cells, Figure 2I) revealed that B and T cells were distributed into a typical follicular and interfollicular organization, suggesting that these patches were intestinal lymphoid structures. We next performed whole mount immunostaining for B220 and CD3 (T cells) and found several structures (1-3) in every $D v l 1^{-{ }^{-}}$mouse, which were preferentially localized to the mid colon (Figure 2, B and E). In addition, the increase in inflammatory cells observed in the $D v l 1^{-/-}$mice was not restricted to the lymphoid structures but was also seen in other regions (Figure 2, C, D, F, and G). Indeed, immunostaining for CD45 (hematopoietic cells) of sections from the ileum and mid colon revealed increased CD45 cells in the small intestine and colon (Supplemental Figure 2, A and B). These findings point to an increase in inflammatory cells throughout the GI tract of $D v l 1^{-1-}$ mice.

To characterize the inflammatory response in the GI tract of the $D v l 1^{-1-}$ mice, we performed flow cytometry of the lamina propria from the ileum and proximal colon of WT and $D v l 1^{-1-}$ mice, gating for lymphoid and myeloid cell populations (Supplemental Figure 3). Analysis of the lymphoid cell populations revealed an increase in the colonic $\mathrm{CD}^{+} \alpha \beta \mathrm{T}$ cells and a trend towards an increase in the ileum (Figure $3 \mathrm{~A}$ ). Immunostaining for CD4 and CD8 showed that both the ileum and mid colon had an increase in CD8 ${ }^{+}$ cells in $D v l 1^{-1-}$ mice (Figure 3B and Supplemental Figure 2C). Next, we wanted to assess the activation state of $\mathrm{CD}^{+} \alpha \beta \mathrm{T}$ cells with flow cytometric analysis. Using the early activation marker of C-type lectin domain family (CD69), we observed an increased number of colonic CD69hi CD8 ${ }^{+} \alpha \beta \mathrm{T}$ cells in $\mathrm{Dvl1}^{-{ }^{--}}$mice with a trend toward an increased number in the ileum (Figure 3C). In addition, CD107a, which marks degranulation and correlates with cytokine secretion and cytotoxic activity in $\mathrm{CD}^{+} \alpha \beta \mathrm{T}$ cells (33), was increased in the colonic $\mathrm{CD}^{+} \alpha \beta \mathrm{T}$ cells of $\mathrm{Dvl1^{-/- }}$ mice with a trend towards an increase in the ileum (Figure 3D). However, the number of $\mathrm{CD}^{+} \alpha \beta \mathrm{T}$ cells that were positive for the long-lived memory $\mathrm{T}$ cell marker interleukin-7 receptor alpha chain (CD127) and the effector T cell marker killer cell lectin-like receptor G1 (KLRG1) did not differ between WT and $\mathrm{Dvl1^{-1- }}$ mice (Figure 3, C and D). To determine if a change in regulatory $\mathrm{T}$ cell number could contribute to the observed increase in $\mathrm{CD} 8^{+} \mathrm{T}$ cells in $\mathrm{Dvl1^{-1- }}$ mice, we performed flow cytometry and gated for FoxP3, Gata3, and ROR $\gamma$ t (Supplemental Figure 3). However, we did not observe a significant change in FoxP3 regulatory $\mathrm{T}$ cells by flow cytometry (data not shown) or immunostaining (Supplemental Figure 2E), suggesting that regulatory $\mathrm{T}$ cells do not play a major role in the inflammatory response observed in $D v l 1^{-/-}$mice.

Flow cytometric analysis of the myeloid cell population revealed an increase in macrophages $\left(\mathrm{F} 4 / 80^{+}\right)$ in the colon of $\mathrm{Dvl1}^{-1-}$ compared to WT mice (Figure 3E). Correspondingly, immunostaining for Iba-1, which marks macrophages, showed a significant increase in Iba- $1^{+}$cells in the colon but not in the ileum of $D v l 1^{-/-}$mice (Figure 3F and Supplemental Figure 2D). To test if the effect of Dvl1 deletion on the immune cells is restricted to the GI tract or also affects other lymphoid organs, we performed flow cytometry on cells from the inguinal and mesenteric lymph nodes and the thymus of $D v l 1^{-1-}$ and WT mice. Analysis of lymphoid organs did not reveal significant changes in the number of lymphoid cells, although the mesenteric lymph nodes had elevated frequencies of both $\mathrm{CD} 69^{\text {hi }} \mathrm{CD} 4^{+}$and $\mathrm{CD} 69^{\mathrm{hi}} \mathrm{CD} 8^{+} \alpha \beta \mathrm{T}$ cells, which presumably reflects drainage to these nodes from the GI tract (Supplemental Figure 2, F and G).

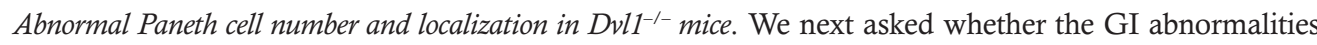
that we observed were associated with defects in the epithelial cells that line the GI tract. To analyze the epithelial cells, we first examined proliferation in the small and large intestine by Ki67 staining. Although the number of $\mathrm{Ki}^{+} 7^{+}$cells was unchanged in the small intestine, it was reduced in the mid colon of $D v l 1^{-1-}$ mutant mice compared to WT mice (Supplemental Figure 4, A and B), consistent with the known role of Wnt signaling as a growth-promoting pathway in the intestine (18). qPCR analysis of the ileum and proximal colon from WT and $D v l 1^{-/-}$mice revealed that $A x i n 2$, a transcriptional readout of canonical Wnt signaling, was reduced in the ileum and proximal colon of $D v l 1^{-/-}$mice (Supplemental Figure 4C). In addition, the epithelial stem cell markers Lgr5 and Ascl2, which are Wnt target genes, were downregulated in the proximal colon and, to a lesser extent, in the ileum of $\mathrm{Dvl1}^{-/-}$mice (Supplemental Figure $4 \mathrm{C}$ ). Interestingly, an additional surrogate marker for the intestinal stem cells, Olfm4, did not change either by qPCR or in situ hybridization analyses (Supplemental Figure 4, C and D). As Olfm4 is thought to be a Notch target gene, these results suggest a specific decrease in Wnt signaling in the stem cells, consistent with the known role of Dvl1 in the Wnt pathway.

It was previously shown that Wnt signals in the crypt can drive Paneth cell maturation (34). Differentiated epithelial cells migrate from the crypt onto the villus, while Paneth cells are normally positioned at the base of the intestinal crypt, where the Wnt signal is highest. Using the Paneth cell 

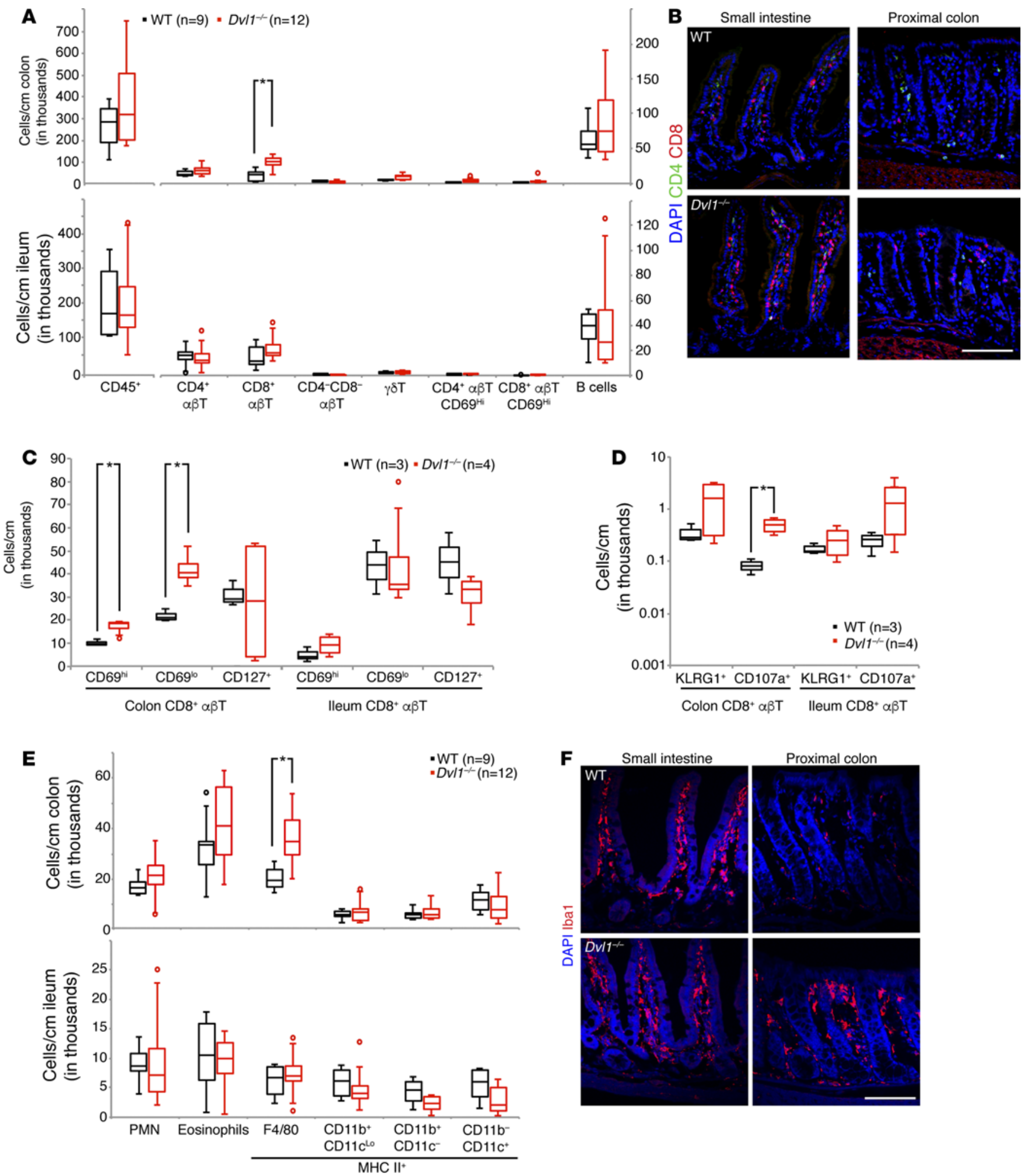

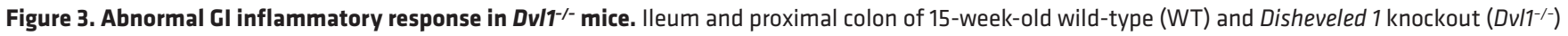
mice were processed for flow cytometry. (A) Lymphoid cell markers were used to determine the number of specific lymphoid cell populations per $\mathrm{cm}$ tissue. Results are presented as box plot, Bonferroni ${ }^{*} P<0.001$ for comparison of the $D v / 1^{-1-}$ mice CD8 $\alpha \beta T$ cells with those from WT. (B) Sections from ileum and proximal colon of WT and Dv/1 / mice were stained with DAPI (nuclei) and immunostained for CD4 and CD8, and representative images are shown. Scale bar: $100 \mu \mathrm{m}$. (C and D) Markers of the activation state of CD8 ${ }^{+} \alpha \beta T$ cells were used to determine the number of activated CD8 ${ }^{+} \alpha \beta T$ cells per $\mathrm{cm}$ tissue. Results are presented as box plot, Bonferroni ${ }^{*} P<0.05$ for comparison of the Dv/1 $7^{-1}$ mice CD8 ${ }^{+} \alpha \beta T$ cells with those from WT. (E) Myeloid cell markers were used to determine the number of specific myeloid cell populations per $\mathrm{cm}$ tissue. Results are presented as box plot, Bonferroni ${ }^{*} P<0.05$ for comparison of

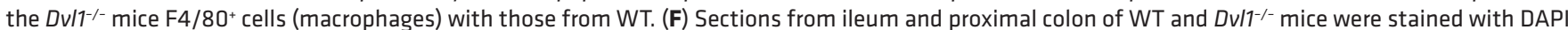
and immunostained for Iba-1 (macrophages), and representative images are shown. Scale bar: $100 \mu \mathrm{m}$. 
A Small intestine

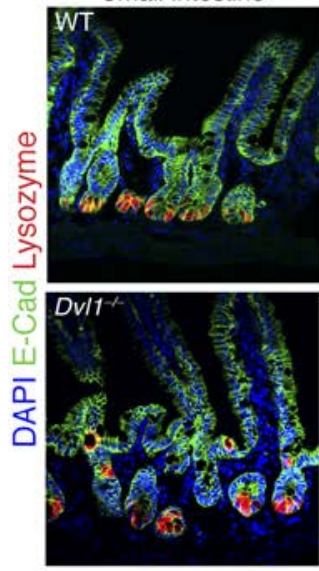

E

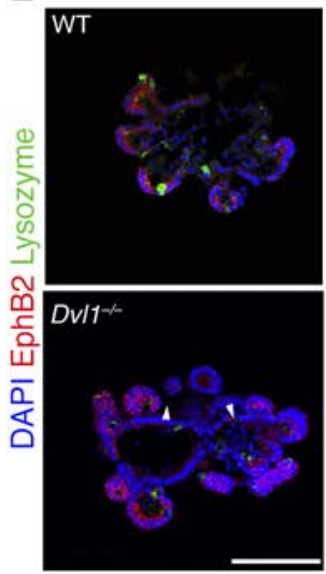

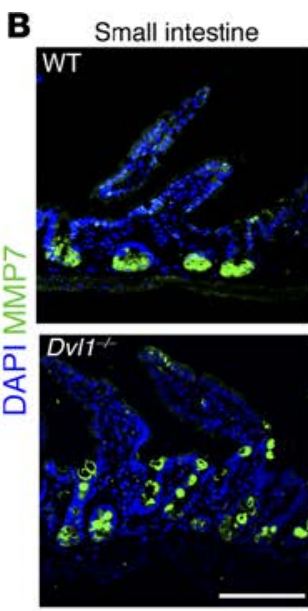

$\mathbf{F}$

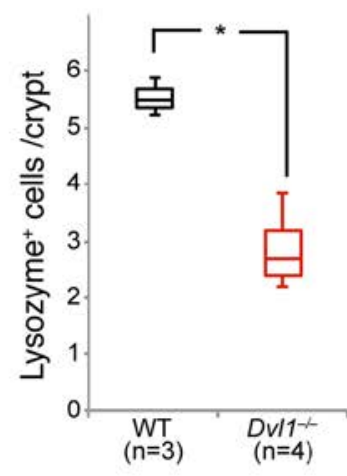

C

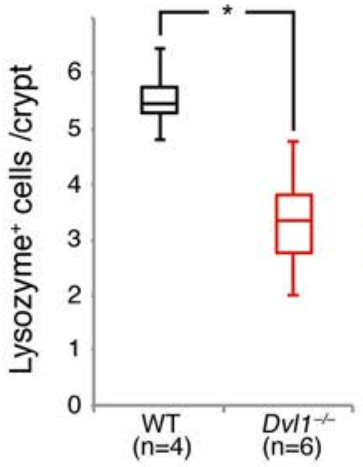

G
D

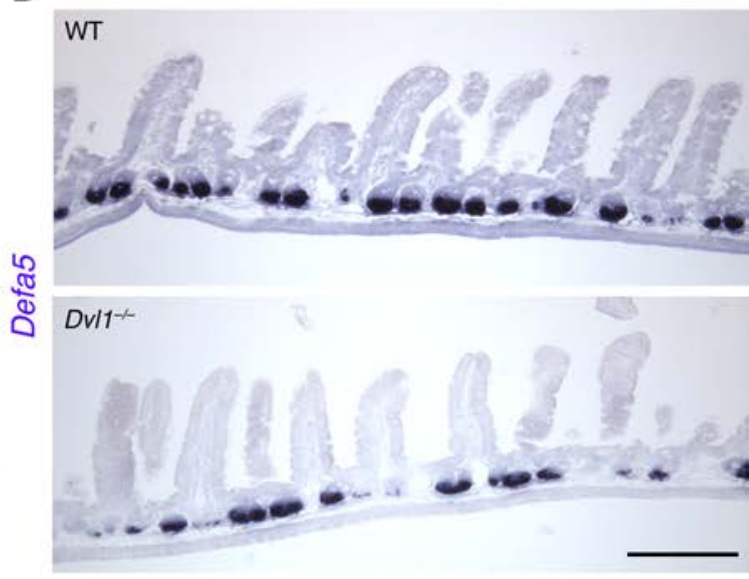

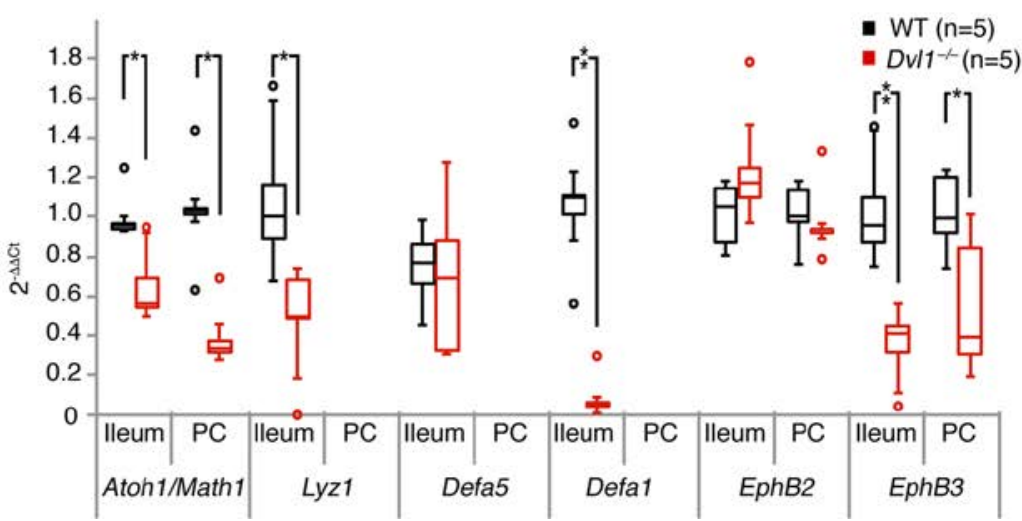

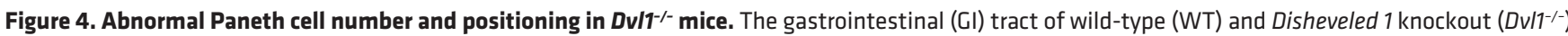
mice were processed for histology, RT-PCR, and in situ hybridization. (A) Sections from the ileum of 12-week-old mice were stained with DAPI (nuclei) and immunostained for E-cadherin and lysozyme, and representative images are shown. (B) Sections from the ileum of 12-week-old mice were stained with DAPI and immunostained for MMP7, and representative images are shown. Scale bar: $100 \mu \mathrm{m}$. (C) Quantification of the number of DAPI+lysozyme ${ }^{+}$cells per crypt are presented as box plot (Bonferroni ${ }^{*} P<0.004$ for comparing the results of the Dv/1 ${ }^{-1-}$ with those of the WT mice). (D) Sections from the GI tract of 8-week-old mice were processed for in situ hybridization and probed for Defa5 and representative images are shown. Scale bar: $200 \mu \mathrm{m}$. (E) Sections from the in vitro gut organoids were stained with DAPI and immunostained for lysozyme and EphB2, and representative images are shown. Scale bar: $100 \mu \mathrm{m}$. (F) Quantification of the numbers of DAPI+lysozyme ${ }^{+E p h B 2}{ }^{+}$cells per crypt are presented as box plot (Bonferroni ${ }^{*} P<0.003$ for comparing the results of the $D v / 1^{-1-}$ with those of the WT mice). (C) RNA from ileum and proximal colon (PC) of 12-week-old WT and Dv/1 $1 /$ mice was extracted and RT-PCR was performed. The levels of Atoh1/Math1, Lyz1, Defa5, Defa1, EphB2, and EphB3 were normalized to Gapdh and are presented as box plot ( $n=5$, Bonferroni ${ }^{*} P<0.05,{ }^{* *} P<0.005$ for comparing the results of the Dv/1 ${ }^{-/-}$with those of the WT mice).

marker lysozyme, we found that the number of Paneth cells was reduced in $D v l 1^{-/-}$mice (Figure 4 , A-C). Moreover, the Paneth cells were not restricted to the base of the crypt in the $D v l 1^{-1-}$ mice, as in WT mice (Figure 4A). These findings were corroborated using a second Paneth cell marker, MMP7 (Figure 4B). In situ hybridization showed that Defa5, which encodes the antimicrobial peptide defensin, was downregulated in the intestinal crypts of the $D v l 1^{-/-}$mice (Figure 4D), and we did not observe mislocalized Defa5 ${ }^{+}$cells in the $D v l 1^{-1-}$ mice. These two observations suggest that the Paneth cells in $D v l 1^{-/-}$mice are less active than in controls.

To determine if the mislocalization and reduction in the number of Paneth cells in $D v l 1^{-1-}$ mice is a cell-autonomous effect of the epithelium, we isolated and cultured crypts from WT and $D v l 1^{-1-}$ mice. In the absence of immune and stromal cells, the isolated crypt epithelium expands to form multicellular $3 \mathrm{D}$ epithelial organoids that mimic the morphology of the adult intestine. Using EphB2 to mark the boundaries of the crypts (35) and lysozyme to mark the Paneth cells, we found a reduced number as well as mislocalization of Paneth cells in $D v l 1^{-/-}$compared to WT intestinal organoids (Figure 4, E and F), thus recapitulating the in vivo findings. These data suggest that the Paneth cell abnormalities in the $D v l 1$ 
A
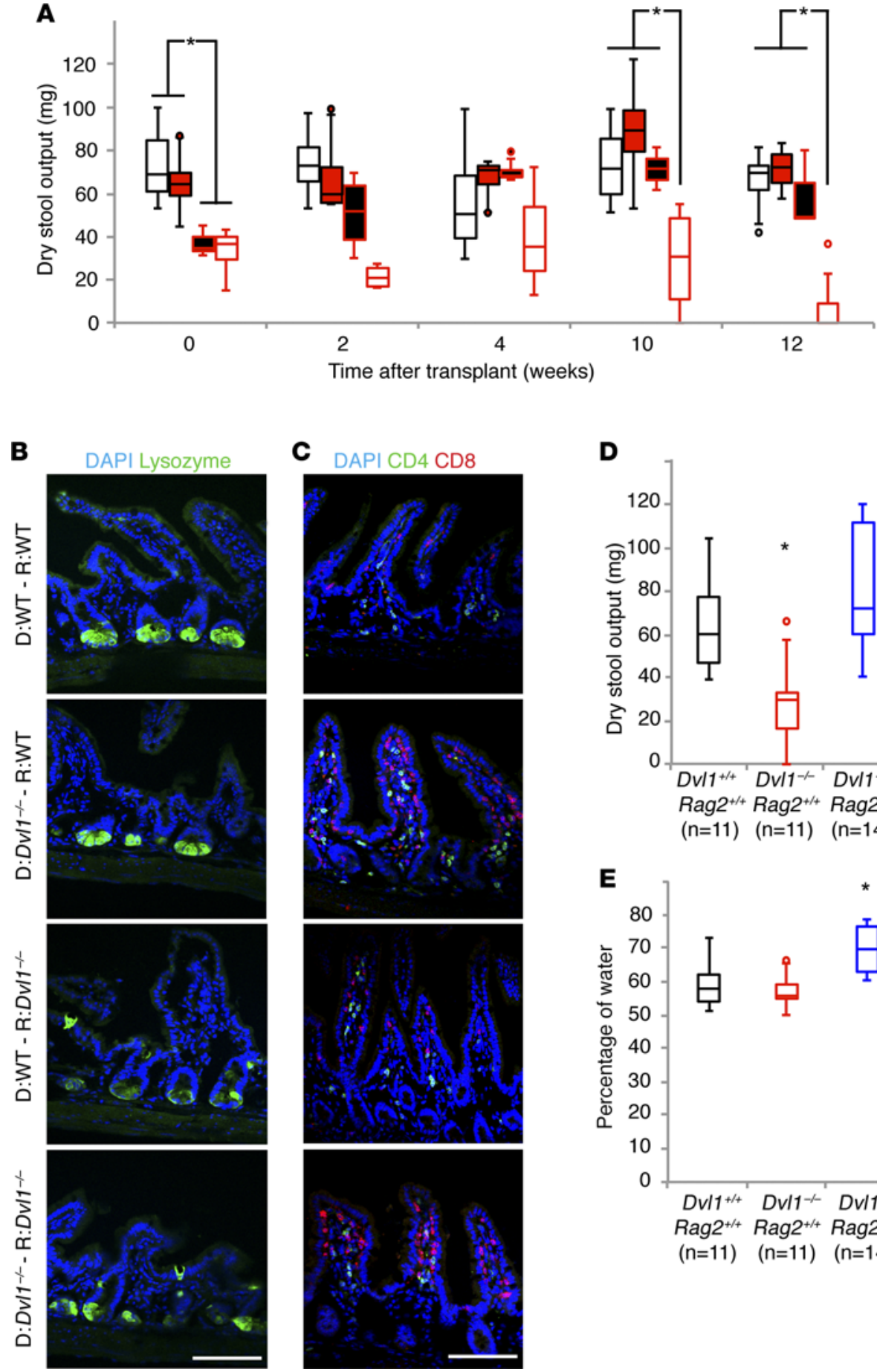

D

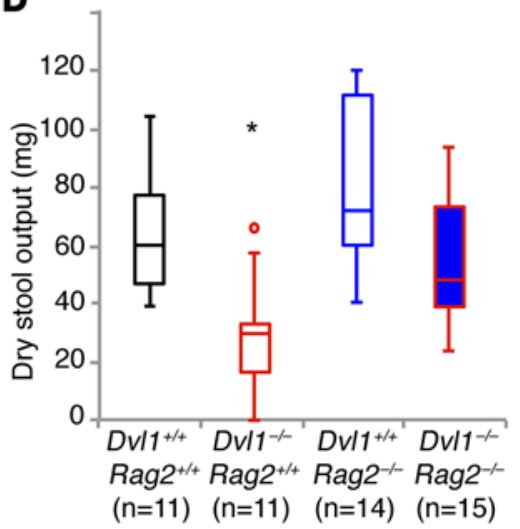

E

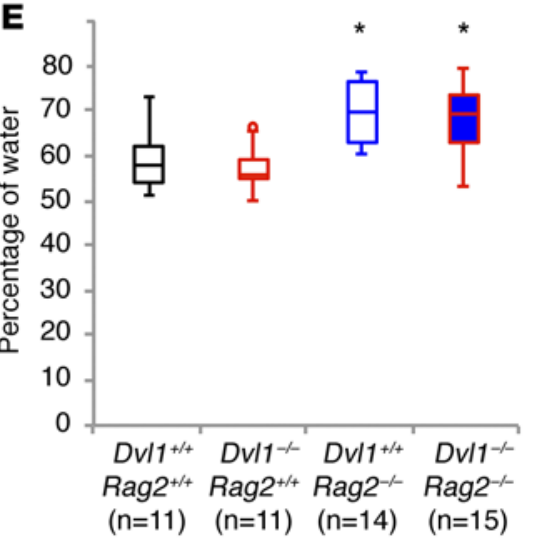

DD:WT R:WT

$\mathrm{D}: D \mathrm{~V} / 1^{-1-\mathrm{R}: \mathrm{WT}}$

D:WT R:Dvl1 ${ }^{-\alpha}$

$\square \mathrm{D}: D v 11^{-1-} \mathrm{R}: D v 11^{-1-}$

Figure 5. T cells are required to induce abnormal GI physiological function. Bone marrow chimera of wild-type (WT) and Disheveled 1 knockout (Dv/1 $\left.1{ }^{--}\right)$mice were prepared by subjecting recipient mice to sublethal doses of radiation and injecting them with hematopoietic progenitor cells from donor mice.

(A) Stool produced over a 1-hour time period was measured and dry stool output was calculated prior to irradiation and following bone marrow chimera preparation (D, donor; R, recipient). Results are presented as box plot, $(n=3$, 2-way ANOVA $P<0.02$, Bonferroni ${ }^{*} P<0.04$ for comparison of the Dvv1 1/- dry stool weight with those from WT). (B and C) The GI tract of WT and $D v / 7^{-1-}$ bone marrow chimera mice was processed for histology and sections were stained with DAPI (nuclei) and immunostained for lysozyme (B), or DAPI, $\mathrm{CD} 4$, and CD8 (C), and representative images are shown. Scale bar: $100 \mu \mathrm{m}$. (D and E) Dv/1/-- mice were backcrossed with Rag2 $^{-/-}$mice and at 8 weeks of age mice were tested for stool produced over a 1-hour time period. Dry stool output (D) and percentage of water in the stool (E) were calculated. Results are presented as box plot, 2-way ANOVA $P<0.05$, Bonferroni ${ }^{*} P<$ 0.05 for comparison of the $D v / 1^{-1}$ dry stool weight with those from all the other mice groups; 2-way ANOVA effect of Rag2 $^{-1-} P<0.001$; ${ }^{* *} P<0.002$ for comparison of the Rag2 $^{-1-}$ and DV/1 $1^{-/-}$Rag2 $^{-/-}$percentage of water in the stool with those from the other mice groups.

mutants are cell autonomous and that the immune compartment is not required to maintain the Paneth cell abnormalities in $D v l 1^{-/-}$mice.

Wnt signaling regulates secretory cell lineage commitment (36) and the position of Paneth cells by controlling the expression of EphB3 (21). Accordingly, RT-PCR analysis of the GI tract of WT and Dvl1 ${ }^{-1}$ mice revealed that the secretory progenitor marker, Atoh1, was downregulated in the GI tract of $D v l 1^{1_{-}}$mice (Figure $4 \mathrm{G}$ ). In addition, in $D v l 1^{-/-}$mice, transcription of the Paneth cell marker, $L y z 1$, was downregulated (Figure 4G), consistent with the reduced Paneth cell number (Figure 4, A-C). Moreover, transcriptional analysis of the Paneth cell antimicrobial peptides Defa5 and Defa1 revealed a trend toward reduction of Defa 5 and a marked decrease in Defa1 (Figure 4G), indicating reduced functional activity of the Paneth 
cells. Lastly, expression of EphB3 was downregulated in the GI tract of $D v l 1^{-1-}$ mice, further demonstrating mispositioning of the Paneth cells in the GI tract of $D v l 1^{-1-}$ mice.

Paneth cells and CD8 cells both contribute to abnormal GI function in Dvl1-1- mice. Our findings indicated both an increase in $\mathrm{CD}^{+} \mathrm{T}$ cells and abnormalities in Paneth cell number and localization. We therefore hypothesized that effects on either the T cells or the Paneth cells individually, or a combined effect on both populations, contributed to the abnormal GI function in $D v l 1^{-1-}$ mice. To examine the role of the inflammatory cells in the GI abnormalities, we performed bone marrow chimera studies, which enabled us to distinguish between the role of the immune (donor) and the epithelial (host) cells. As we had previously observed, dry stool output was decreased in $D v l 1^{-1-}$ mice prior to initiating bone marrow chimera studies (Figure 5A). Consistent with what we had previously observed, $D v l 1^{-1-}$ mice that were reconstituted with Dvl1 $1^{-1}$ bone marrow had decreased stool output compared with WT mice that were reconstituted with WT bone marrow (compare Figure 1A and Figure 5A). Interestingly, WT bone marrow that was reconstituted into $D v l 1^{-/-}$mice rescued the stool output phenotype, indicating that provision of a normal immune compartment ameliorates the GI dysfunction in $D v l 1$-mutant mice. However, $D v 11^{-/-}$bone marrow reconstituted into WT mice could not induce the reduced dry stool output phenotype, demonstrating that both immune and epithelial cells are required for induction of the GI dysfunction. In regards to possible involvement of the irradiation-resistant compartment, because the WT bone marrow rescued $D v l 1^{-1}$ mice, this suggests that the irradiation-resistant compartment does not play a major role in the phenotype.

Immunostaining for lysozyme revealed that $D v l 1^{-1-}$ mice that received either WT or $D v l 1^{-1-}$ bone marrow had reduced and mislocalized Paneth cells (Figure 5B), regardless of the genotype of the bone marrow donor, supporting our in vitro findings that Paneth cell abnormalities in $D v l 1^{-1-}$ mice are not affected by the immune cells (Figure 4, E and F). In addition, double labeling of CD4 and CD8 revealed that WT and $D v l 1^{-1-}$ mice that received bone marrow from $D v l 1^{-1-}$ mice had increased CD8 ${ }^{+}$cells (Figure $5 \mathrm{C}$ ), indicating that the bone marrow reconstitution indeed formed a host-immune chimera and that deletion of $D v l 1$ directly affects $\mathrm{T}$ cell maturation and/or differentiation. The bone marrow chimera experiments demonstrated that the Paneth cell phenotype in $D v 11^{-/-}$mice is not regulated by the increase in $\mathrm{CD} 8^{+} \mathrm{T}$ cells, and also that the Paneth cell dysfunction does not result in the increase of $\mathrm{CD} 8^{+} \mathrm{T}$ cells. Taken together, these findings indicate that abnormalities in both the immune and epithelial cells are required to induce the observed gut dysfunction.

We hypothesized that the increase in $\mathrm{CD}^{+} \mathrm{T}$ cells plays an important role in the GI function of $D v l 1^{-1}$ mice. To test this hypothesis, we crossed $D v l 1^{-1-}$ mice with $R a g 2^{-1-}$ mice, which lack B and T cells, and found

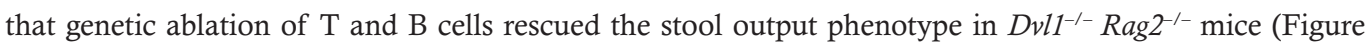

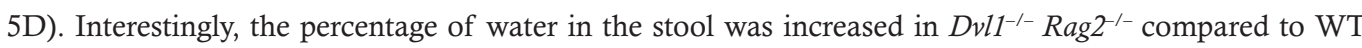

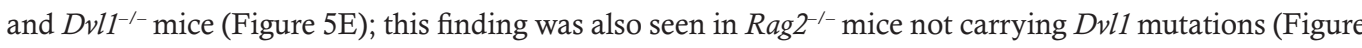
$5 \mathrm{E})$. Thus, deletion of Rag2 rescued the reduced dry stool output that was observed in $D v l 1^{-1-}$ mice. Collectively, these findings support the notion that $\mathrm{CD} 8^{+} \mathrm{T}$ cells play an important role in the abnormal GI physiology of $D v l 1^{-/-}$mice.

Paneth and $C D 8^{+} T$ cells converge on regulation of microbial composition in the GI tract of Dvl1 ${ }^{-1-}$ mice. Because absence of $D v l 1$ in both immune cells and epithelial cells was required to induce the GI dysfunction, we hypothesized that the aberrant immune and epithelial cells act together on the GI microbiome, which ultimately causes the physiological abnormalities. To examine this, we first compared bacterial microbiota of WT and $D v l 1^{-/-}$animals using $16 \mathrm{~S}$ rRNA-based sequencing. Six-week-old $D v l 1^{-{ }^{-}}$mice exhibited a significant decrease in ileal alpha diversity indices (phylotype richness, Pielou's evenness, and Faith's phylogenetic diversity) compared to WT controls, although this decrease did not persist at 12 weeks of age (Figure 6, A-C, and Supplemental Figure 6, A-C). Ileal microbiota composition (beta diversity) of 6-weekold $D v l 1^{-/-}$mice further supported these observations and was significantly different (weighted UniFrac PERMANOVA, $r^{2}=0.24, P=0.018$ ) from that of age-matched WT mice (Figure 6D). As was observed for alpha indices, beta diversity did not significantly differ between WT and $D v l 1^{-/-}$groups at 12 weeks of age (Supplemental Figure 6D).

In the mid colon, no significant differences in alpha diversity were observed between the $D v l 1^{-{ }^{-}}$and age-matched WT mice at either 6 or 12 weeks of age (Supplemental Figure 6, E-G and I-K). However, significant differences in beta diversity were observed at both time points using an unweighted UniFrac distance matrix (unweighted UniFrac PERMANOVA, $r^{2}=0.19, P=0.002$ ) (Supplemental Figure 6, H and L). These data indicate that the nature of the dysbiosis associated with absence of $D v l 1$ is distinct in 
A

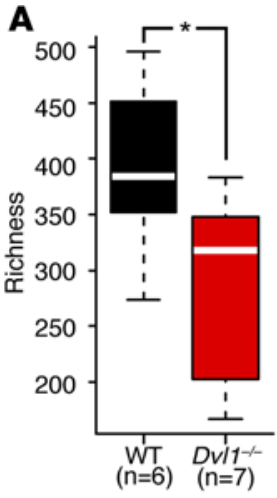

B

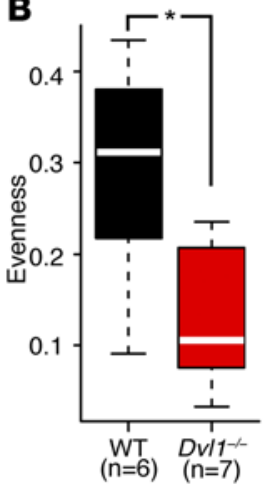

C

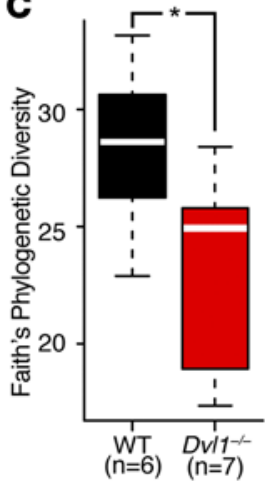

D

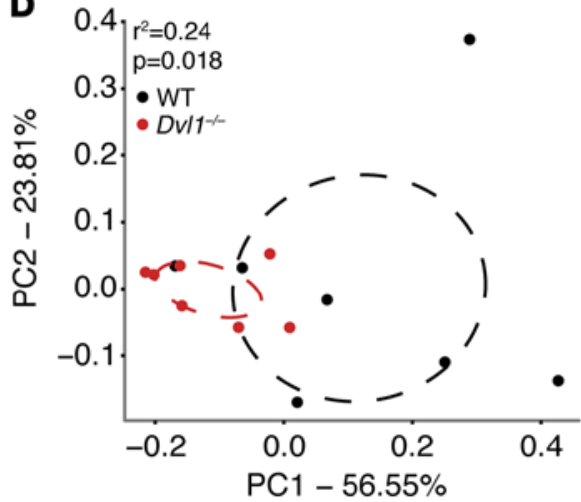

$\mathbf{F}$

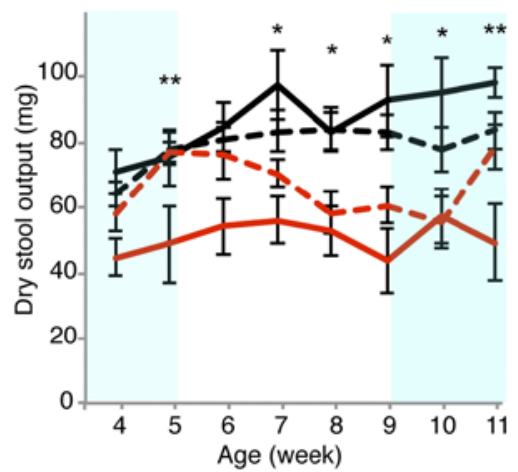

$\rightarrow$ WT SH $(\mathrm{n}=8) \quad-D v / 1^{-<} \mathrm{SH}(\mathrm{n}=8)$

$-\leadsto-$ WT CH $(n=12) \quad-\infty-D v l 1^{-/} \mathrm{CH}(\mathrm{n}=12)$
G

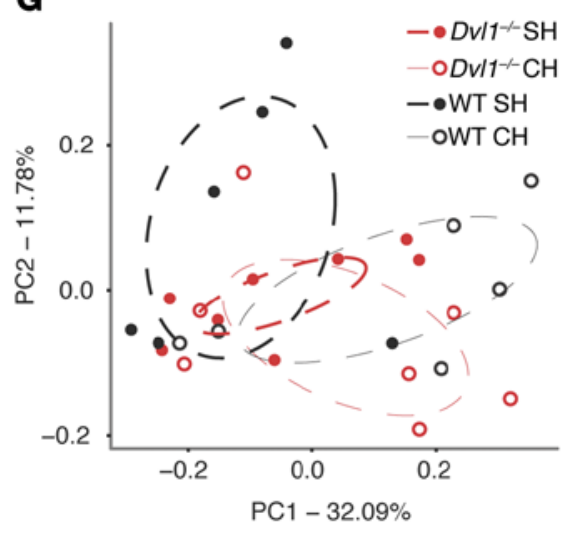

H
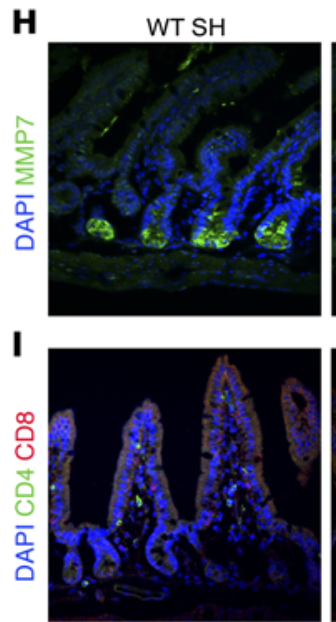

WT CH
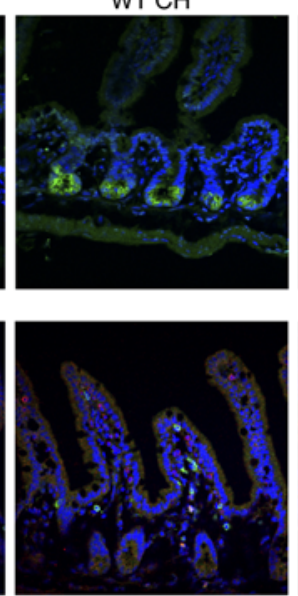

$D v / 1^{-1} \mathrm{SH}$
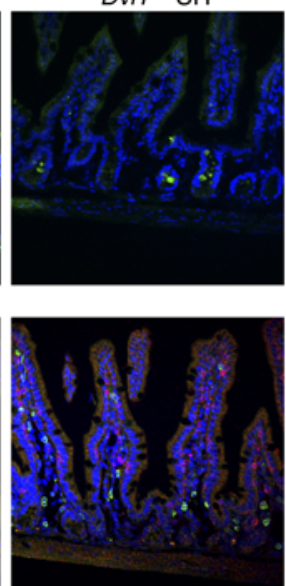
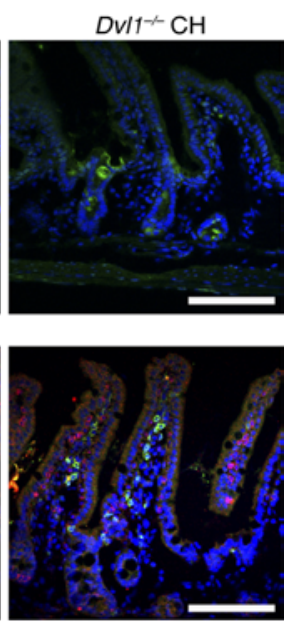

Figure 6. Abnormal microbiome composition in Dv/1//- mice. Ileum and proximal colon of wild-type (WT) and Disheveled 1 knockout (Dv/1-/-) mice were processed for 165 rRNA gene profiling. (A-C) Ileum of 6-week-old WT and Dv/1/- mice 16S rRNA gene profiling of alpha diversity indices (phylotype richness, Pielou's evenness, and Faith's phylogenetic diversity) was analyzed compared to WT mice. Results are presented as box plots, ${ }^{*} P<0.05$ for comparing the results of the Dv/1//- with those of the WT mice. (D) 165 rRNA gene profiling of beta diversity from ileum of individual 6-week-old WT and Dv/1 ${ }^{-/-}$mice using weighted UniFrac distance matrix is presented. Permanova statistical analysis revealed significant differences $(P=0.019)$ in microbiota composition of Dv/1 1/- and WT mice. (E) Relative abundance of Helicobacter OTU (10961) and Flexispira OTU (1141335) in the ileum and mid colon of 6- and 12-week-old WT and $D v / 1^{-/-}$mice was measured. Results are presented as box plots. ${ }^{*} q<5.0 \times 10^{-2},{ }^{* *} q<5.0 \times 10^{-5}$, ${ }^{* *} q<5.0 \times 10^{-11}$ for comparing the results of $D v / 1^{-/-}$mice with age-matched WT mice. (F) WT and Dv/1 ${ }^{-1-}$ mice were cohoused (CH) or standardly housed (SH) by genotype from the day of weaning. $\mathrm{CH}$ and SH mice stool produced over a 1-hour time period was measured weekly and dry stool output was calculated. Results are presented as mean \pm SEM. ${ }^{*}<0.01$ for 2-way ANOVA effect by genotype; ${ }^{* *} P<0.03$ for 2 -way ANOVA effect by genotype $\times$ housing; and Bonferroni $P<0.04$ for comparison of the $D v / 1^{-1-}$ SH dry stool weight with those from all other mouse groups. (G) 165 rRNA gene profiling of beta diversity from ileum of individual 12-week-old WT and $D v / 1^{-/-}$mice using unweighted UniFrac distance matrix is presented. Permanova statistical analysis revealed significant difference $(P=0.036)$ in microbiota composition

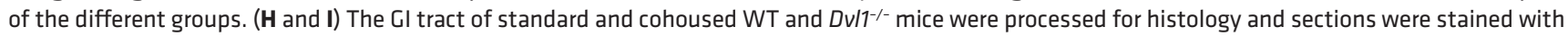
DAPI (nuclei) and immunostained for MMP7 (H), or DAPI, CD4, and CD8 (I), and representative images are shown. Scale bar: $100 \mu \mathrm{m}$. 
different GI compartments. Whereas differences in ileal bacterial communities of $D v l 1^{-/-}$and WT mice are primarily driven by relative abundances of phylogenetically distinct bacteria, colonic community differences are associated with the presence or absence of distinct taxa. Collectively, these findings suggest that $D v l 1^{-1-}$ mice exhibit distinct early-life ileal and colonic microbiota perturbations, leading us to hypothesize that these differences play a mediating role in the onset and persistence of the abnormal GI phenotype.

Next, we sought to identify the specific taxa that discriminated ileal communities of $D v 1^{-/-}$mice from those of WT animals (see Methods for details). Sixteen taxa consisting of members of Helicobacteraceae, S24-7, Streptococcaceae, Lachnospiraceae, Coriobacteriaceae, Ruminococcaceae, and Pseudomonadaceae and the order Clostridiales were identified as significantly enriched in the ileum of $D v l 1^{-1-}$ mice at 6 weeks compared to age-matched controls (Supplemental Table 1). A substantial degree of overlap was evident between those taxa significantly enriched in the ileum and mid colon of $D v l 1^{-1-}$ mice at 6 weeks (Supplemental Table 1). However, a greater number of taxa exhibited significant differences in relative abundance in the mid colon $(n=40)$, and the difference in relative abundance was more profound in this compartment (Supplemental Table 1). At 12 weeks of age, ileal and colonic communities of $D v l 1^{-/-}$mice also exhibited overlap in the taxa that were significantly enriched (Supplemental Table 1). Of note, three specific taxa, including Helicobacter (OTU 10961) and Flexispira (OTU 1141335), were consistently significantly enriched in both the ileum and colon of $D v l 1^{-1-}$ mice at 6 and 12 weeks of age (Figure 6E). This indicates that the physiological and immunological changes associated with $D v l 1$ deletion promote enrichment of these microbiota members in both the ileum and colonic compartments of the GI tract and implicated them in the associated GI dysfunction.

Since ileal and colonic gut microbiota perturbations characterized $D v l 1^{-/-}$mice, we cohoused these mice with WT to determine whether microbial transfer between mice, due to coprophagia, could rescue the abnormal GI physiological function. $D v l 1^{-/-}$mice that were continuously cohoused with WT mice from weaning until 5 weeks of age exhibited normal dry stool output (Figure 6F). In addition, in the previously cohoused $D v l 1^{-1-}$ mice, separation into standard housing resulted in reversion, within 3 weeks, of the GI phenotype. Moreover, cohousing WT and $D v l 1^{-/-}$mice a second time rescued the dry stool output in the $D v l 1^{-/-}$mice again, implicating a transferrable microbial mediator in abrogating the GI dysfunction in $D v l 1^{-/-}$animals.

Analysis of the ileal bacterial community of the re-cohoused mice indicated that compositional changes in the gut microbiome occurred at this site upon cohousing (Figure 6G). Immunostaining for lysozyme revealed that regardless of cohousing status, $D v l 1^{-1-}$ mice exhibited sustained Paneth cell mislocalization (Figure $6 \mathrm{H}$ ), suggesting that loss of $D v l 1$ rather than perturbation of the microbiota determines the Paneth cell abnormalities in $\mathrm{Dvl1^{-/- }}$ mice. In addition, double labeling of CD4 and CD8 revealed that $\mathrm{Dvl1^{-{ } ^ { - - } }}$ mice had increased $\mathrm{CD}^{+}$cells compared to WT mice, regardless of the housing conditions (Figure 6I). These data further support the notion that the increase in $\mathrm{CD}^{+} \mathrm{T}$ cells is a result of the absence of $D v l 1$ and not perturbation of the microbiota. Taken together, these findings suggest that genetically induced host abnormalities in both immune and epithelial cells are upstream of the microbiota perturbations.

In-depth taxonomic examination of both cohoused and standardly housed $D v l 1^{-/-}$mice revealed that rescue of the abnormal GI function in the cohoused mice was associated with significant enrichment of unclassified members of the Bacteroidales and multiple taxa belonging to the genus Lactobacillus, among others (Supplemental Table 2). A Lactobacillus taxon was amongst the most highly enriched taxa, having a genus level classification in both the ileum and colon of the $D v l 1^{-/-}$cohoused mice. We have previously shown that oral supplementation of a member of this genus, Lactobacillus johnsonii, (isolated from murine cecum) alters ileal microbiota composition and reduces proinflammatory responses (37). We therefore isolated the dominant culturable Lactobacillus from these mice by plating serial dilutions of stool on Man Rogosa Sharpe (MRS) agar. The complete 16S rRNA gene from 10 randomly chosen colonies was PCR amplified and sequenced for species level identification. Basic Local Alignment Search Tool (BLAST) analysis indicated that 9 of the 10 colonies displayed identical 16S rRNA gene sequences and shared the highest homology to L. johnsonii.

We next examined whether supplementation of $D v 1^{-/-}$mice with $L$. johnsonii impacted their GI function and ileal microbiome composition. WT and $D v l 1^{-1-}$ mice (6 weeks old) were supplemented orally 3 times per week for 3 weeks with L. johnsonii isolated from the $D v l 1^{-1-}$ cohoused mice, and dry stool output was measured. Following 2 weeks of $L$. johnsonii supplementation, $D v l 1^{-1-}$ mice dry stool output was rescued to WT levels (Figure 7A). Analysis of ileal beta diversity of WT, $D v l 1^{-/-}$mice, and $D v l 1^{-/-}$mice supplemented with $L$. johnsonii 
A

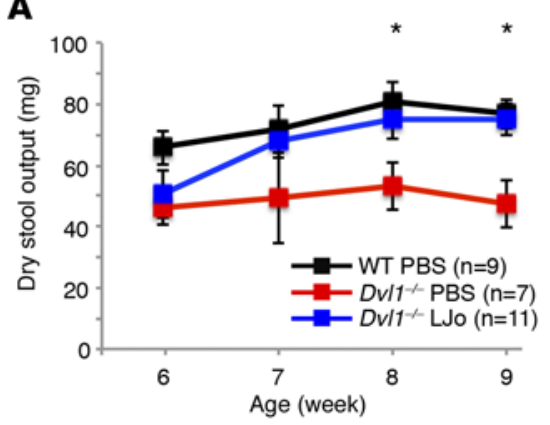

B

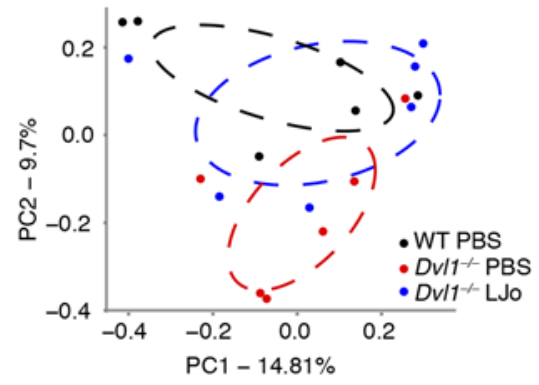

D

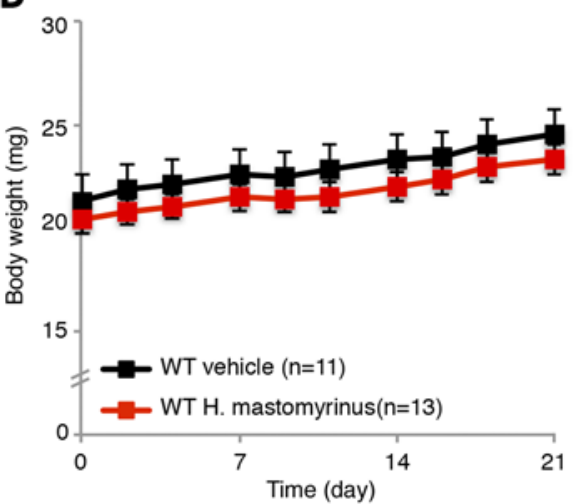

$\mathbf{C}$

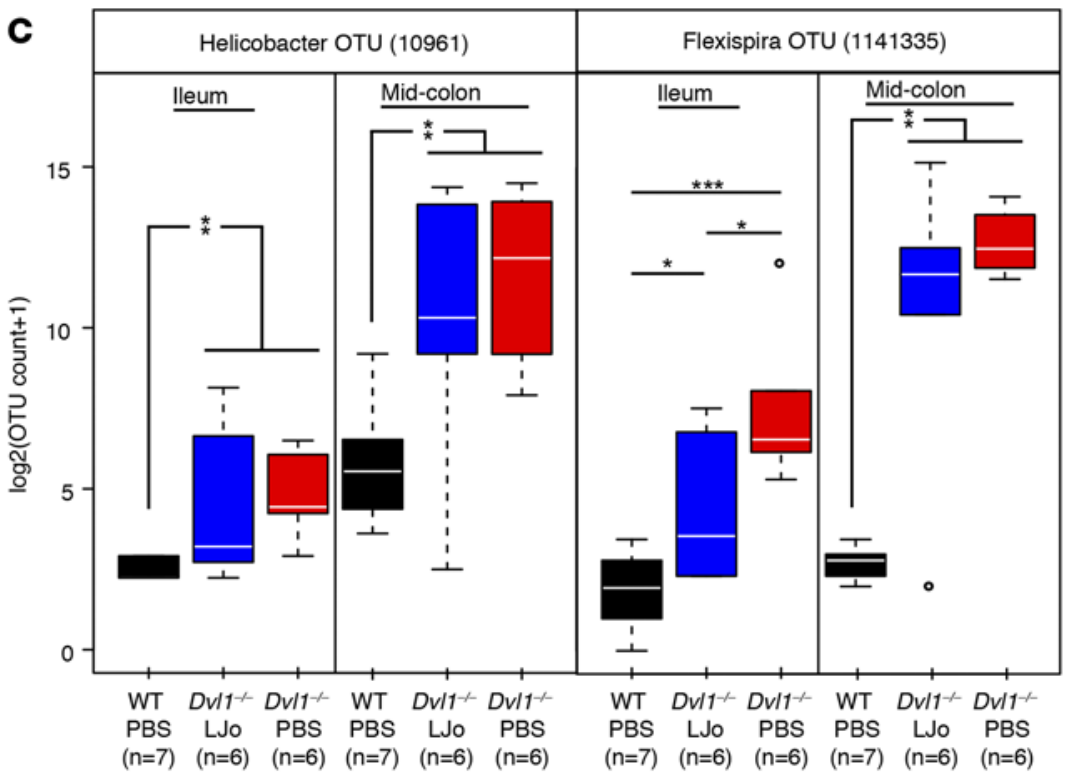

$\mathbf{E}$

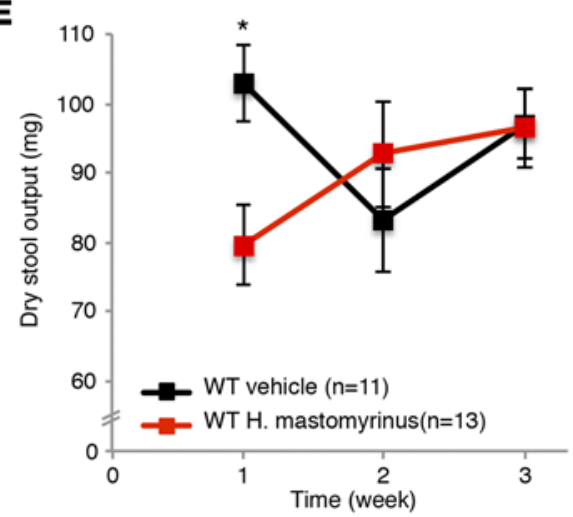

$\mathbf{F}$

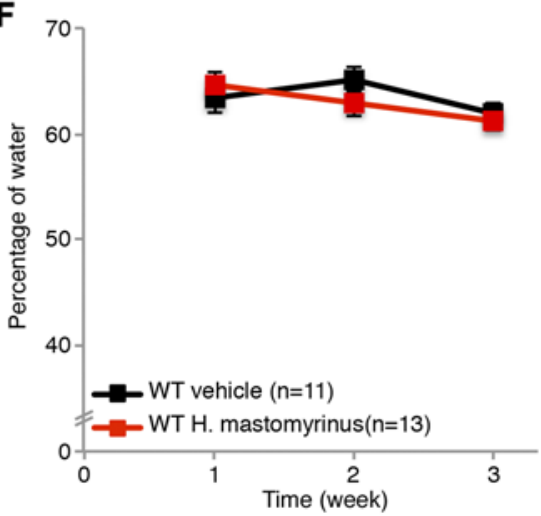

Figure 7. Manipulation of the microbiota regulates GI function. (A-C) Six-week-old wild-type (WT) and Disheveled 1 knockout (Dv/1 $\left.{ }^{-/-}\right)$mice were fed with either PBS or Lactobacillus johnsonii (LJo) $\left(1.5 \times 10^{9} \mathrm{CFU}\right) 3$ times per week for 3 weeks. (A) Stool produced over a 1-hour time period was measured weekly, and dry stool output was calculated. Results are presented as the mean $\pm \mathrm{SEM},\left(P<0.03\right.$ for ANOVA, Bonferroni ${ }^{*} P<0.05$ for comparison of the $D v / 1^{-/-}$ PBS-fed stool weight with those from WT PBS-fed and Dv/1-/- LJo-fed mice). (B) 165 rRNA gene profiling of beta diversity of the individual mice using an unweighted UniFrac distance matrix is presented. (C) Relative abundance of Helicobacter OTU (10961) and Flexispira OTU (1141335) in the ileum and mid colon of PBS- and LJo-fed mice was measured. Results are presented as box plots, ${ }^{*} q<8.0 \times 10^{-3},{ }^{* *} q<3.0 \times 10^{-4}$, ${ }^{* *} q<2.0 \times 10^{-10}$. (D-F) Six-week-old WT mice were fed with either vehicle or Helicobacter mastomyrinus (108 CFU) 3 times per week for 3 weeks. (D) Body weight was measured before each feeding. Results are presented as the mean \pm SEM. (E) Stool produced over a 1-hour time period was measured weekly, and dry stool output was calculated. Results are presented as the mean \pm SEM (Bonferroni ${ }^{*} P<0.008$ for comparison of the WT Helicobacter mastomyrinus-fed mice dry stool output weight with those from WT vehicle-fed mice). (F) Stool produced over a 1-hour time period was measured weekly, and percentage of water content in the stool was calculated. Results are presented as the mean \pm SEM.

indicated that supplementation altered the composition of the microbiota at this site, resulting in an intermediate bacterial composition between that of WT and $\mathrm{Dvll}^{-/-}$mice (Figure 7B). More specifically, a taxonomic comparison of $D v l 1^{-1-}$ mice supplemented with L. johnsonii or PBS revealed that the taxon that exhibited the greatest degree of depletion in ileal communities of the supplemented mice was the same Flexispira taxon that was consistently enriched in $\mathrm{Dvll}^{--}$mice (compared to WT controls) at 6 and 12 weeks of age (Figures $6 \mathrm{E}$ and $7 \mathrm{C}$ ).

We hypothesized that expansion of members of the Flexispira taxon (OTU 1141335) in the GI tract may play a major role in the GI dysfunction of $\mathrm{Dvll}^{-/-}$mice. To address this, we next sought to isolate 
Flexispira OTU 1141335 members from Dvl1 ${ }^{-/-}$animals, and we identified Helicobacter mastomyrinus as a member of this taxon (98\% sequence identity with the dominant 16S rRNA of Flexispira OTU 1141335). $H$. mastomyrinus was therefore used to orally supplement WT mice (6 weeks old) 3 times per week for 3 weeks with $10^{8} \mathrm{CFU}$ of this species. Body weight, dry stool output, and water stool content were measured. Throughout the feeding experiment, the gross appearance and behavior of animals was normal, and no change in weight was observed between vehicle- and $H$. mastomyrinus-supplemented WT mice (Figure 7D). However, following 1 week of $H$. mastomyrinus supplementation, the dry stool output of supplemented animals was reduced compared to vehicle-treated mice (Figure 7E). Importantly, the reduced stool output did not persist following 2 and 3 weeks of feeding, despite sustained supplementation of $H$. mastomyrinus, suggesting that compensatory immune or microbial mechanisms in these animals led to restoration of the normal phenotype. The water content in the stool was not different between $H$. mastomyrinus-supplemented mice compared with nonsupplemented WT mice (Figure 7F), suggesting a specific effect of $H$. mastomyrinus on the GI motility phenotype in $D v l 1^{-/-}$mice.

\section{Discussion}

In this study, we have shown that epithelial and immune dysfunction in the GI tract of Dvl1-mutant mice is associated with an abnormal microbiome that contributes to aberrant GI function. Initially, we set out to determine whether loss of Dvll function affected GI function. We found that dry stool output was reduced and correlated with an increase in WGTT in Dvl1 mutants compared to controls. We next found an aberrant immune response in the $D v l 1^{-/-}$mice that was characterized by an increase in $\mathrm{CD}^{+} \alpha \beta \mathrm{T}$ cells throughout the GI tract and an increase in colonic macrophages compared to WT mice. Examination of the epithelial compartment revealed a reduction in number and mislocalization of Paneth cells in the Dvll mutant. Using bone marrow chimeras, we found that both immune and epithelial cells are required to induce the GI dysfunction, leading us to hypothesize that the aberrant immune and epithelial cells act together on the GI microbiome, which ultimately causes the physiological abnormalities. Indeed, we found altered microbiota composition in Dvl1-mutant mice, and although cohousing $D v l 1^{-/-}$with WT mice led to compositional changes in the GI microbiome and rescued the gut transit phenotype, it did not rescue either the immune or epithelial cell phenotypes. Moreover, oral supplementation of $D v l 1^{-1-}$ animals with L. johnsonii, an organism significantly enriched in cohoused $D v l 1^{-{ }_{-}}$animals with restored normal gut transit, rescued this phenotype. This was associated with a significant decrease in the relative abundance of a specific Flexispira taxon (OTU 1141335) that also demonstrated the greatest degree of relative enrichment in both the ileum and colon of 6- and 12-week-old $D v l 1^{-1-}$ mice. Indeed, oral supplementation of WT animals with $H$. mastomyrinus isolated from $D v 1^{-1-}$ animals and belonging to the Flexispira OTU 1141335 taxon induced acute GI dysfunction following 1 week of oral supplementation to WT animals. Collectively, these data indicate that the immune dysfunction that occurs in $D v l 1^{-1-}$ animals results in an altered gut microenvironment that facilitates overgrowth of opportunistic pathogens and depletion of organisms that promote normal gut function. Importantly, the Dvl-associated gut transit abnormalities could be rescued by microbial manipulation of the gastrointestinal microbiome.

Intestinal immunity is regulated by Dvl1. Our finding of increased CD $8^{+} \alpha \beta \mathrm{T}$ cells in the Dvl1 mutant (Figure 3) raises the question of whether the Dvll mutation directly affects the T cells or if this is an indirect effect caused by the microbiota perturbation. It was previously shown that Helicobacter infection induces $\mathrm{CD}^{+} \mathrm{T}$ cell responses in pigs (38), which suggests that the $\mathrm{CD} 8^{+}$induction in $\mathrm{Dvl1^{-/ }}$ mice was due to increased opportunistic bacterial pathogen enrichment caused by abnormal Paneth cell function (Figure 4). However, the fact that WT mice reconstituted with Dvl1-mutant bone marrow displayed an increased number of $\mathrm{CD}^{+} \mathrm{T}$ cells, but with normal Paneth cells, suggests that loss of $D v l 1$ has an intrinsic effect on the hematopoietic compartment. In addition, Dvl1-mutant mice that were cohoused with WT mice had altered bacterial microbiota but the $\mathrm{CD} 8^{+} \alpha \beta \mathrm{T}$ cell number did not change, providing further support for a direct effect of the Dvl1 mutation on the immune phenotype. Indeed, the phenotype may be intrinsic to $\mathrm{CD}^{+} \mathrm{T}$ cells, as Wnt signaling affects multiple stages of $\mathrm{T}$ cell differentiation and proliferation (39). Conflicting findings were reported for the role of Wnt signaling during the transition of double-positive into single-positive (CD4 or CD8) T cells in the thymus. On the one hand, it was shown that a gain-of-function mutant of $\beta$-catenin preferentially increased $\mathrm{CD}^{+} \mathrm{T}$ cells (40), but in another study, $\beta$-catenin gain-of-function mice were reported to improperly respond to TCR-dependent 
positive selection signals (41-43). These findings may suggest that the level of $\beta$-catenin and the developmental stage at which $\beta$-catenin is expressed influence how Wnt signaling is read out.

Activated cytotoxic $\mathrm{CD} 8^{+} \mathrm{T}$ cells are vital in defense against viruses and intracellular bacteria. Activated effector $\mathrm{CD} 8^{+} \mathrm{T}$ cells secrete cytokines, whereas memory $\mathrm{CD} 8^{+} \mathrm{T}$ cells provide enhanced protection against the same pathogen. $\beta$-Catenin was shown to have the potential to regulate mature $\mathrm{CD} 8^{+} \mathrm{T}$ cell function via several mechanisms (44-46). Importantly, activation of $\beta$-catenin facilitated $\mathrm{CD} 8^{+}$memory $\mathrm{T}$ cell formation, with enhanced protective capacity and extended survival of $\mathrm{CD} 4{ }^{+} \mathrm{CD} 25^{+}$regulatory $\mathrm{T}$ cells (47). Thus, it is possible that in Dvl1-mutant mice the increase in $\mathrm{CD} 8^{+} \mathrm{T}$ cells is a result of reduced capability to transition into memory $\mathrm{T}$ cells. Future studies will be needed to address the direct mechanism by which $\beta$-catenin signaling affects $\mathrm{T}$ cell development and function.

Wnt signaling regulates Paneth cell differentiation and function. Our finding of reduced number and mislocalization of Paneth cells in the Dvll mutant (Figure 4) is strongly supported by multiple studies focused on the effect of Wnt signaling on Paneth cells. Mucosal Wnt signaling in adult intestine is confined to the crypt, including progenitor and Paneth cells $(34,48)$. In addition, Wnt signaling maintains the undifferentiated state of intestinal crypt progenitor cells and also induces maturation of Paneth cells in intestinal crypts (49). Our finding of mislocalized Paneth cells in Dvll-mutant mice is similar to previously identified phenotypes in Wnt signaling-related mutants (34) and EphB knockout mice (34). The Wnt signaling gradient controls expression of the EphB/ephrinB sorting receptors and ligands. These receptors were shown to regulate the correct positioning of Paneth cells at the bottom of the crypt (21). These data suggest that misregulation of EphB3 in Dvl1 mutants (Figure 4G) induces mislocalization of Paneth cells along the villus-crypt axis.

Paneth cells secrete antimicrobial peptides such as defensins and lysozyme to control the microbial content of the intestine. In addition, it was recently shown that TCF-1-mediated Wnt signaling activates $\alpha$-defensin transcription (50). These results also support our finding of reduced defensin transcription in Dvl1 mutants (Figure 4, D and G). Paneth cells not only control populations of potentially pathogenic organisms, but also modulate the endogenous bacterial communities of the gut. Accordingly, the important impact of host genotype on Paneth cell function was shown to regulate the composition of the intestinal microbiota (51). Taken together, our findings suggest that aberrant Paneth cell function results in a failure to control GI pathogens, including Flexispira and Helicobacter, and results in altered gut bacterial community composition.

Immune cells contribute to normal microbiota colonization. Previous work has shown that the immune cells and the microbiome regulate each other in a bidirectional manner. Experiments in germ-free mice demonstrated that the microbiota regulates the development of acquired immunity (52), and that the host plays an important role in selecting its particular microbiota (53). It was previously suggested that the immune system discriminates between pathogens and the microbiota through recognition of symbiotic bacterial molecules in a process that prompts commensal colonization (54). Based on these studies and the findings reported here, we suggest that the increase in $\mathrm{CD} 8^{+} \alpha \beta \mathrm{T}$ cells in the GI tract of $D v 11$ mutants misregulates commensal colonization, which contributes to the abnormal function. The majority of the literature focuses on the role of $\mathrm{CD}^{+}$regulatory $\mathrm{T}$ cells in shaping the intestinal microbiota (55), but our data do not support a major role for the $\mathrm{CD}^{+}$regulatory $\mathrm{T}$ cells in our system (Supplemental Figure 2E). The pathogenic role of $\mathrm{CD}^{+} \mathrm{T}$ cells in inflammatory bowel disease (IBD) has been shown using animal models and implicated in human IBD patients. CD8 ${ }^{+} \mathrm{T}$ cells can escape both central and peripheral tolerance and may trigger autoimmune reactions to a microbial mimic of self-antigen (12). Several in vivo studies have supported the role of pathogenic $\mathrm{CD} 8^{+} \mathrm{T}$ cells in response to self- or exogenous antigens in the intestinal mucosa (13, $14,56)$. It is possible that loss of $D v l 1$ may affect antigen-presenting cells, which could induce an aberrant $\mathrm{T}$ cell response due to 'misreading' of the commensal bacteria. Thus, the specific mechanism by which $\mathrm{CD} 8^{+}$ $\mathrm{T}$ cells are misregulated and in turn misregulate the microbiota will require additional studies.

Microbiome composition regulates GI motility. Our findings suggest that abnormalities in both the CD8 ${ }^{+} \mathrm{T}$ cells and Paneth cells are required to induce gut dysfunction in Dvll-mutant mice. Abnormalities in these constituents of the intestinal ecosystem regulate the microbiota composition, which in turn regulates GI motility. It was recently shown that the microbiota can regulate host physiology (57). Elimination of the microbiota caused dilatation of the cecum as a result of abnormal motility that returned to normal upon recolonization with bacteria (58), and it has been proposed that the mechanism underlying this involves regulation by the microbiota of Toll-like receptor expression in the intestine (59). Conversely, decreased 
motility of the GI tract can lead to changes in the microbiota community composition (60-62). However, the rescue of the GI dysfunction by cohousing and L. johnsonii supplementation suggests that in our system the microbiome is upstream of GI function. We identified a Flexispira (OTU 1141335) that was significantly enriched in the GI tract of $D v l 1^{-1-}$ mice (Figure $6 \mathrm{E}$ ) and was significantly depleted following L. johnsonii supplementation of $\mathrm{Dvl1}^{-/-}$mice, suggesting that this Helicobacteraceae plays a central role in the regulation of GI motility. Indeed, oral supplementation of $H$. mastomyrinus to WT mice acutely induced GI dysfunction akin to that observed in $D v l 1^{-1-}$ mice (Figure 7E). The mechanism by which this microbe exerts its effect on GI motility is still unclear, and future studies will be needed to address the mechanisms and pattern of interactions with the enteric nervous system that induce the abnormal GI motility.

\section{Methods}

Mice. WT and $D v l 1^{-1-}$ mice were generated on the 129S6/SvEvTaconic background. Genotyping for the

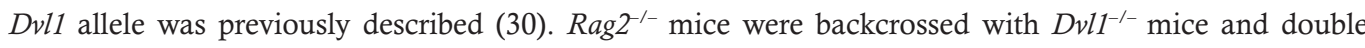

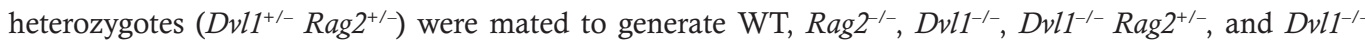
Rag $2^{-/-}$littermates. The histological experimental data from each genotype were generated from at least 3 litters. Each biochemical/flow experiment was performed on 2 or 3 cohorts of mice. The behavioral experiments were performed on 5 cohorts of mice and 4 times throughout the study. Mice were housed in standard cages and maintained on a $12: 12$-hour light/dark cycle at $22^{\circ} \mathrm{C}$. All testing occurred during the light portion of the cycle.

Metabolic cages. Twelve-week-old mice were singly housed and measurements were taken every 12 minutes for 4 days, including oxygen consumption, carbon dioxide output, food consumption, water consumption, and 3 unique measures of movement. The respiratory exchange ratio (RER) and heat were calculated as $\mathrm{VCO}_{2} /$ $\mathrm{VO}_{2}$ and $\mathrm{VO}_{2}(3.815+1.232 \times \mathrm{RER})$, respectively. Heat, $\mathrm{VO}_{2}$, and $\mathrm{VCO}_{2}$ were all normalized to effective body mass $\mathrm{Vxx}=\mathrm{Vxx} /[\text { weight }(\mathrm{g}) / \text { mass unit }]^{0.75}$, per the manufacturer's recommendations. Stool produced was collected every day at the same time of day (12-1 PM) and weighed. Stool was then desiccated at $75^{\circ} \mathrm{C}$ overnight to determine dry weight. Stool water was calculated as the difference between these two measurements.

Stool weight. Total stool weight was measured by placing the mice in a novel environment and collecting all stool produced over a 1-hour time period at the same time of day (10-11 AM). Stool was then desiccated at $75^{\circ} \mathrm{C}$ overnight to determine dry weight. Stool water is the difference between these two measurements.

Colonic motility. Colonic motility was measured by the bead expulsion test as previously described (63). Briefly, a glass bead (diameter, $3 \mathrm{~mm}$ ) was inserted through the anus and pushed, with a polished glass rod, into the colon for a distance of $2 \mathrm{~cm}$. The time required for expulsion of the glass bead was recorded.

Whole gut transit time. WGTT was measured by oral gavage of $0.3 \mathrm{ml}$ of $6 \%(\mathrm{w} / \mathrm{v})$ carmine dye in $0.5 \%$ methylcellulose (Sigma-Aldrich) to each mouse as previously described (64). The time taken from administration of carmine until the first appearance of one red pellet was recorded.

Metabolic testing. Whole-animal metabolic analysis was performed by the UCSF Mouse Metabolism Core using CLAMS (Comprehensive Laboratory Animals Monitoring System) as per the manufacturer's instructions (Columbus Instruments) cages.

Flow cytometry. For isolation of intestinal lamina propria for flow cytometry, the large intestine and small intestine were dissected. The mesenteric fat and Peyer's patches were removed. The proximal $1 / 2$ of large intestine and distal 1/3 of small intestine were used for cell isolation. Intestinal pieces were inverted and shaken in three changes of $20 \mathrm{ml}$ cold PBS. Tissues were washed for 20 minutes at $37^{\circ} \mathrm{C}$ in two changes of $20 \mathrm{ml} \mathrm{Ca}{ }^{2+} / \mathrm{Mg}^{2+}$-free HBSS containing $5 \mathrm{mM}$ DTT, $5 \mathrm{mM}$ EDTA, $10 \mathrm{mM} \mathrm{HEPES}$, and 2\% FCS, followed by $20 \mathrm{ml}$ of $\mathrm{Ca}^{2+} / \mathrm{Mg}^{2+}$-replete HBSS containing $10 \mathrm{mM}$ HEPES with $2 \% \mathrm{FCS}$. Tissues were digested for 30 minutes at $37^{\circ} \mathrm{C}$ in $5 \mathrm{ml} \mathrm{Ca}{ }^{2+} / \mathrm{Mg}^{2+}$-replete HBSS containing $10 \mathrm{mM} \mathrm{HEPES,} \% \mathrm{FCS}, 30 \mu \mathrm{g} / \mathrm{ml}$ DNase, $0.1 \mathrm{Wünsch} / \mathrm{ml}$ LibTM (Roche), and homogenized in C tubes using a gentleMACS tissue dissociator (Miltenyi). Homogenate was passed through a $100-\mu \mathrm{m}$ filter, separated in a $40 \% / 90 \%$ Percoll gradient, and enumerated for staining equivalent numbers for flow cytometry. The following antibodies (from BioLegend, eBioscience, and BectonDickinson) were used for staining: CD4 (RM4-5), CD8a (53-6.7), CD8b (YTS156.7.7), CD11b (M1/70), CD11c (N418), CD45 (30-F11), CD69 (H1.2F3), CD90.2 (53-2.1), CD107a (eBio1D4B), CD127 (A7R34), F4/80 (BM8), Foxp3 (FJK-16s), gdTCR (GL3), IgA (C10-3), KLRG1 (2F1/ KLRG1), Ly6C (HK1.4), Ly6G (1A8), MHCII (M5/114.15.2), SigF (E50-2440), TCRb (H57). For intracellular staining, cells were first labeled with LIVE/DEAD fixable dye (Invitrogen) and then, following staining for extracellular antigens, cells were fixed and permeabilized with the Foxp3 Staining Buffer Set 
(eBioscience) for staining of intracellular antigen. The gating strategy is depicted in Supplemental Figure 2.

Bone marrow chimera. Twelve-week-old 129S6/SvEvTaconic and $D v l 1^{-1-}$ mice were irradiated with $1,000 \mathrm{rad}$. The following day, irradiated mice were sedated with isoflurane and injected retro-orbitally with $5 \times 10^{6}$ bone marrow cells obtained from 2 femurs and 2 tibias of 8-week-old 129S6/SvEvTaconic or $D v l 1^{-1-}$ mice.

Histology and immunohistochemistry. The intestine was flushed and stretched out with $4 \%$ paraformaldehyde (PFA) after cardiac perfusion on a cold glass plate. Alternating 2-cm pieces were collected in 2 cassettes. One cassette was fixed overnight with $4 \%$ PFA, followed by a $30 \%$ sucrose incubation overnight at $4^{\circ} \mathrm{C}$. Tissues were embedded in frozen O.C.T. compound (Tissue-Tek) and sectioned at $7 \mu \mathrm{m}$. The other cassette was fixed overnight with 4\% PFA and processed for paraffin embedding and sectioned at $2 \mu \mathrm{m}$. Sections were treated identically and staining was performed on the same day for all genotypes. Cresylviolet/Nissl and immunohistochemistry staining were performed by standard protocols. For immunohistochemistry, sections were air dried for 1 hour and then rehydrated in PBS followed by incubation with blocking solution (5\% donkey serum and 0.5\% Triton X-100 in PBS) for 1 hour at room temperature (RT). Sections were incubated with primary antibodies diluted in blocking solution for 24 hours at $4^{\circ} \mathrm{C}$, followed by the appropriate secondary antibody conjugated with Alexa Fluor 488, 568, or 647 (Molecular Probes Invitrogen). Primary antibodies used were rat anti-CD4 (100532 BioLegend), rat anti-CD8 (100727 BioLegend), rat anti-CD45 (103109 BioLegend), rat anti-B220 (RM2620 Life Technologies), rat anti-CD3 (100240 BioLegend), rabbit anti-Iba-1 (019-19741 Wako), rat anti-FoxP3 (FJK-16s eBioscience), rabbit anti-Ki67 (SP-6 Thermo Scientific), rat anti-E-cadherin (13-1900 Invitrogen), rabbit anti-lysozyme (A0099 Dako), and goat anti-MMP7 (AF2967 R\&D Systems). After immunohistochemistry, slides were mounted with DAPI counterstain (Invitrogen, P36930).

Primary mouse small intestinal epithelial cell cultures. Intestinal crypts were isolated from 6-week-old 129S6/SvEvTaconic and $D v l 1^{-/-}$mice according to a previously published protocol (65). The proximal half of the mouse small intestine was opened longitudinally, washed with ice-cold PBS, and villi were scraped off using a coverslip. Intestine was cut into 2- to 4-mm pieces and crypts were isolated using $2 \mathrm{mM}$ EDTA in PBS at $4^{\circ} \mathrm{C}$ for 30 minutes. Crypts were found to be enriched in fractions 4-6 and they were combined and filtered through a $70-\mu \mathrm{m}$ cell strainer. About 500 crypts were plated into a $50-\mu 1$ Matrigel (BD Biosciences) droplet. The Matrigel droplet was supplemented with crypt culture medium (Advanced DMEM/ F12 (Life Technologies) supplemented with 2 mM GlutaMax (Life Technologies), $10 \mathrm{mM} \mathrm{HEPES,} \mathrm{and} 100$ $\mathrm{U} / \mathrm{ml}$ penicillin/100 $\mathrm{mg} / \mathrm{ml}$ streptomycin, N2 supplement $(1 \times)$, B27 supplement $(1 \times), 1 \mathrm{mM} N$-acetylcysteine, $50 \mathrm{ng} / \mathrm{ml} \mathrm{EGF,} 100 \mathrm{ng} / \mathrm{ml}$ Noggin, and $1 \mathrm{mg} / \mathrm{ml}$ R-spondin), and the medium was replaced every 4 days and cultures were replated every 14 days. In preparation for immunostaining, intestinal crypt cultures were fixed in 4\% PFA for 10 minutes at room temperature, samples were transferred to a $30 \%$ sucrose solution for 24 hours at $4^{\circ} \mathrm{C}$, embedded, and flash frozen in O.C.T. compound (Tissue-Tek). Frozen coronal sections $(10 \mu \mathrm{m})$ were then cut on a cryostat and mounted on Super-frost slides and stored at $-80^{\circ} \mathrm{C}$ until use.

$R T-q P C R$. Total cellular RNA was extracted with TRIzol (Invitrogen) and purified in a column using the RNeasy Mini Kit (QIAGEN). DNAase treatment was performed in the column. Extracted RNA $(1 \mu \mathrm{g})$ samples were reverse transcribed into cDNA according to the manufacturer's protocol for RTPCR (Bio-Rad, 170-8891). qPCR was performed on $10 \mathrm{ng}$ cDNA by using SsoFast EvaGreen Supermix (Bio-Rad) and an Eppendorf Mastercycler EP Realplex 2 thermal cycler. The specific primers used were: EphB2, TGCTGCTGCCGCTGCTAGC and TCGTAGCCGCTCACCTCTTCC; EphB3, GCTACACCTTTGAGGTGCAGGC and GTTGTACGGAGTTCTTCTGGC. The following predesigned primer assays from IDT were also used: Axin2 (Mm.PT.53a.8726473), Lgr5 (Mm.PT.58.12492947), Ascl2 (Mm.PT.53a.30838854), Olfm4 (Mm.PT.58.14228836), Atoh1/Math1 (Mm.PT.56a.29687973.g), Lyz1 (Mm.PT.58.7374112), Gapdh (Mm.PT.39a.1).

Image acquisition and analysis. The immunofluorescent slides were viewed and photographed with $10 \times$, $40 \times$, or $60 \times$ objectives using a Nikon C1si microscope. Analysis of the thickness and/or cell counts of immunohistochemical staining were captured and quantified across the entire image taken by the ImagePro Plus system (version 5.1; Media Cybernetics). For each staining, 3 or 4 randomly chosen regions were analyzed per GI region (ileum or colon) corresponding to 15-30 crypts per mouse.

$16 S$ rRNA gene profiling. DNA was purified from tissue samples using the QIAGEN AllPrep DNA/RNA Mini Kit according to the manufacturer's recommendations. The V4 region of the 16S rRNA gene was PCR amplified in triplicate reactions using barcoded primers amenable to Illumina sequencing (66). Triplicate reac- 
tions were combined, purified using the SequalPrep Normalization Plate Kit (Invitrogen), and pooled in approximately equimolar concentrations. Pooled libraries were sequenced in a $251 \times 151 \mathrm{bp}$ sequencing run on the MiSeq Desktop Sequencer (Illumina). Downstream processing of sequencing reads was performed as follows: paired sequencing reads were assembled using FLASh (67) followed by quality filtering and demultiplexing using QIIME (68). Open reference OTU picking was used to cluster demultiplexed sequencing reads at 97\%, chimeras were identified using ChimeraSlayer (69) and removed, and taxonomy was assigned based on the Greengenes database (70). The NCBI Sequence Read Archive (SRA) accession number for our study is SRP076689.

Very low abundance OTUs having less than 1/1000th of a percent of the total read count were removed and the resulting OTU table was rarefied to a depth of 76,079 reads per sample and further filtered based on the samples being compared.

Diversity calculations. Richness (observed taxa) and evenness were calculated using the vegan (71) package in R (http://www.R-project.org/) and alpha diversity was calculated using the Faith's phylogenetic diversity metric (72). Beta diversity was measured using the weighted and unweighted UniFrac metrics (73) and PERMANOVA $P$ values (adonis) were assigned using the QIIME compare_categories.py script. Principal coordinates analysis (PCoA) plots were generated in R using the ggplot2 package (74).

Identification of significantly enriched taxa. Significantly enriched taxa were identified using a multi-model approach based on earlier research (75). Specifically, Poisson, negative binomial, and zero-inflated negative binomial regression approaches were used to model the count of each OTU and the best model amongst the three approaches was selected using the Bayesian information criterion (BIC). Only OTUs having a false discovery correct $P$ value of less than 0.05 were retained and declared as significantly enriched taxa.

Isolation and identification of supplemented taxa. Lactobacillus spp. were isolated by plating serial dilutions of stool on selective MRS agar and incubating at $37^{\circ} \mathrm{C}$ for 48 hours under anaerobic conditions. The 16S rRNA gene from 10 randomly chosen isolates was PCR amplified using the $27 \mathrm{~F}$ and $1492 \mathrm{R}$ primer set (76), and sequenced using the Sanger method. For taxon identification, BLAST analysis was performed. The dominant L. johnsonii isolate was stored in sterile $20 \%$ glycerol at $-80^{\circ} \mathrm{C}$ until use. L. johnsonii was routinely cultured in MRS broth at $37^{\circ} \mathrm{C}$ for 24 hours, and cells were pelleted and washed in sterile PBS prior to oral gavage.

H. mastomyrinus BAA-1046 was purchased from the American Type Culture Collection and was grown on tryptic soy agar slants with $5 \%$ defibrinated sheep blood under microaerophilic conditions for 3 days. Cells were pelleted and resuspended in sterile PBS prior to oral gavage.

Statistics. All statistical analyses were performed using SPSS version 12. The 4 groups (WT and $D v l 1^{-/-}$) were compared by 1-way ANOVA or 2-way ANOVA for the bone marrow chimera, cohousing, and Lactobacillus feeding experiments. Where appropriate, 2-tailed Student's $t$ test post-hoc analysis was performed with Bonferroni correction for multiple comparisons and the $P$ values $(<0.05)$ presented are those of the corrected values. Data are presented either as mean \pm SEM or as box plot.

Study approval. All animal care and experiments were performed under protocols approved by the University of California, San Francisco Institutional Animal Care and Use Committee.

\section{Author contributions}

HB designed and performed experiments, interpreted results, and wrote the manuscript. AKS performed flow cytometry and bone marrow chimera experiments. DF, DL, and RV performed the 16S rRNA gene profiling. YMK worked on the GI function testing. YN performed in situ hybridization. AWB participated in conception of the study. SVL supervised the $16 \mathrm{~S}$ rRNA gene profiling and participated in design and interpretation of the results. RML supervised the flow cytometry and bone marrow chimera experiments and participated in design and interpretation of the results. ODK supervised the study, designed experiments, and wrote and revised the manuscript.

\section{Acknowledgments}

We are grateful to Julia Schanin, Bradford Youngblood, the members of the Klein and Locksley laboratories, and Averil Ma for their helpful suggestions and advice. This research was supported by funding from the California Institute for Regenerative Medicine (RN3-06525) and NIH (U01-DK103147), both to O.D. Klein.

Address correspondence to: Ophir Klein, 513 Parnassus Avenue, HSE1508, Box 0422, San Francisco, California 94143-0422, USA. Phone: 415.476.4719 or 415.476.4720; E-mail: ophir.klein@ucsf.edu. 
1. Simon-Assmann P, Turck N, Sidhoum-Jenny M, Gradwohl G, Kedinger M. In vitro models of intestinal epithelial cell differentiation. Cell Biol Toxicol. 2007;23(4):241-256.

2. Gerbe F, et al. Distinct ATOH1 and Neurog3 requirements define tuft cells as a new secretory cell type in the intestinal epithelium. J Cell Biol. 2011;192(5):767-780.

3. Dupont HL. Review article: evidence for the role of gut microbiota in irritable bowel syndrome and its potential influence on therapeutic targets. Aliment Pharmacol Ther. 2014;39(10):1033-1042.

4. Koslowski MJ, Beisner J, Stange EF, Wehkamp J. Innate antimicrobial host defense in small intestinal Crohn's disease. Int J Med Microbiol. 2010;300(1):34-40.

5. Ayabe T, Satchell DP, Wilson CL, Parks WC, Selsted ME, Ouellette AJ. Secretion of microbicidal alpha-defensins by intestinal Paneth cells in response to bacteria. Nat Immunol. 2000;1(2):113-118.

6. Cash HL, Whitham CV, Behrendt CL, Hooper LV. Symbiotic bacteria direct expression of an intestinal bactericidal lectin. Science. 2006;313(5790):1126-1130.

7. Hooper LV, Stappenbeck TS, Hong CV, Gordon JI. Angiogenins: a new class of microbicidal proteins involved in innate immunity. Nat Immunol. 2003;4(3):269-273.

8. Vaishnava S, Behrendt CL, Ismail AS, Eckmann L, Hooper LV. Paneth cells directly sense gut commensals and maintain homeostasis at the intestinal host-microbial interface. Proc Natl Acad Sci USA. 2008;105(52):20858-20863.

9. Hadis $\mathrm{U}$, et al. Intestinal tolerance requires gut homing and expansion of $\mathrm{FoxP}^{+}$regulatory $\mathrm{T}$ cells in the lamina propria. Immunity. 2011;34(2):237-246.

10. Murai $\mathrm{M}$, et al. Interleukin 10 acts on regulatory $\mathrm{T}$ cells to maintain expression of the transcription factor Foxp3 and suppressive function in mice with colitis. Nat Immunol. 2009;10(11):1178-1184.

11. Coccia M, et al. IL-1 $\beta$ mediates chronic intestinal inflammation by promoting the accumulation of IL-17A secreting innate lymphoid cells and CD4(+) Th17 cells. J Exp Med. 2012;209(9):1595-1609.

12. Zehn D, Bevan MJ. T cells with low avidity for a tissue-restricted antigen routinely evade central and peripheral tolerance and cause autoimmunity. Immunity. 2006;25(2):261-270.

13. Hacini-Rachinel $\mathrm{F}$, et al. $\mathrm{CD} 4^{+} \mathrm{T}$ cells and Lactobacillus casei control relapsing colitis mediated by $\mathrm{CD} 8^{+} \mathrm{T}$ cells. J Immunol. 2009;183(9):5477-5486.

14. Nancey S, et al. CD8 ${ }^{+}$cytotoxic T cells induce relapsing colitis in normal mice. Gastroenterology. 2006;131(2):485-496.

15. Cadigan KM, Nusse R. Wnt signaling: a common theme in animal development. Genes Dev. 1997;11(24):3286-3305.

16. Moon RT, Brown JD, Torres M. WNTs modulate cell fate and behavior during vertebrate development. Trends Genet. 1997;13(4):157-162.

17. Van der Flier LG, et al. The intestinal Wnt/TCF signature. Gastroenterology. 2007;132(2):628-632.

18. Andreu P, et al. A genetic study of the role of the Wnt/beta-catenin signalling in Paneth cell differentiation. Dev Biol. 2008;324(2):288-296.

19. Koslowski MJ, et al. Genetic variants of Wnt transcription factor TCF-4 (TCF7L2) putative promoter region are associated with small intestinal Crohn's disease. PLoS ONE. 2009;4(2):e4496.

20. Bastide P, et al. Sox9 regulates cell proliferation and is required for Paneth cell differentiation in the intestinal epithelium. J Cell Biol. 2007;178(4):635-648.

21. Batlle E, et al. Beta-catenin and TCF mediate cell positioning in the intestinal epithelium by controlling the expression of EphB/ephrinB. Cell. 2002;111(2):251-263.

22. Vidrich A, et al. Fibroblast growth factor receptor-3 regulates Paneth cell lineage allocation and accrual of epithelial stem cells during murine intestinal development. Am J Physiol Gastrointest Liver Physiol. 2009;297(1):G168-G178.

23. Pongracz J, Hare K, Harman B, Anderson G, Jenkinson EJ. Thymic epithelial cells provide WNT signals to developing thymocytes. Eur J Immunol. 2003;33(7):1949-1956.

24. Blank U, Karlsson G, Karlsson S. Signaling pathways governing stem-cell fate. Blood. 2008;111(2):492-503.

25. Okamura RM, Sigvardsson M, Galceran J, Verbeek S, Clevers H, Grosschedl R. Redundant regulation of T cell differentiation and TCRalpha gene expression by the transcription factors LEF-1 and TCF-1. Immunity. 1998;8(1):11-20.

26. Staal FJ, et al. Wnt signaling is required for thymocyte development and activates Tcf-1 mediated transcription. Eur J Immunol. 2001;31(1):285-293.

27. Verbeek S, et al. An HMG-box-containing T-cell factor required for thymocyte differentiation. Nature. 1995;374(6517):70-74.

28. Ding Y, Shen S, Lino AC, Curotto de Lafaille MA, Lafaille JJ. Beta-catenin stabilization extends regulatory T cell survival and induces anergy in nonregulatory T cells. Nat Med. 2008;14(2):162-169.

29. Lehtonen A, et al. Gene expression profiling during differentiation of human monocytes to macrophages or dendritic cells. J Leukoc Biol. 2007;82(3):710-720.

30. Etheridge SL, et al. Murine dishevelled 3 functions in redundant pathways with dishevelled 1 and 2 in normal cardiac outflow tract, cochlea, and neural tube development. PLoS Genet. 2008;4(11):e1000259.

31. Hamblet NS, et al. Dishevelled 2 is essential for cardiac outflow tract development, somite segmentation and neural tube closure. Development. 2002;129(24):5827-5838.

32. Wang J, et al. Dishevelled genes mediate a conserved mammalian PCP pathway to regulate convergent extension during neurulation. Development. 2006;133(9):1767-1778.

33. Aktas E, Kucuksezer UC, Bilgic S, Erten G, Deniz G. Relationship between CD107a expression and cytotoxic activity. Cell Immunol. 2009;254(2):149-154.

34. van Es JH, et al. Wnt signalling induces maturation of Paneth cells in intestinal crypts. Nat Cell Biol. 2005;7(4):381-386.

35. Wang F, et al. Isolation and characterization of intestinal stem cells based on surface marker combinations and colony-formation assay. Gastroenterology. 2013;145(2):383-395.e1.

36. Ireland $\mathrm{H}$, et al. Inducible Cre-mediated control of gene expression in the murine gastrointestinal tract: effect of loss of betacatenin. Gastroenterology. 2004;126(5):1236-1246.

37. Fujimura KE, et al. House dust exposure mediates gut microbiome Lactobacillus enrichment and airway immune defense 
against allergens and virus infection. Proc Natl Acad Sci USA. 2014;111(2):805-810.

38. Kronsteiner B, Bassaganya-Riera J, Philipson N, Hontecillas R. Novel insights on the role of CD8 ${ }^{+} \mathrm{T}$ cells and cytotoxic responses during Helicobacter pylori infection. Gut Microbes. 2014;5(3):357-362.

39. Yu Q, Sharma A, Sen JM. TCF1 and beta-catenin regulate T cell development and function. Immunol Res. 2010;47(1-3):45-55.

40. Mulroy T, Xu Y, Sen JM. beta-Catenin expression enhances generation of mature thymocytes. Int Immunol. 2003;15(12):1485-1494.

41. Gounari F, et al. Somatic activation of beta-catenin bypasses pre-TCR signaling and TCR selection in thymocyte development. Nat Immunol. 2001;2(9):863-869.

42. Guo Z, et al. Beta-catenin stabilization stalls the transition from double-positive to single-positive stage and predisposes thymocytes to malignant transformation. Blood. 2007;109(12):5463-5472.

43. Xie H, Huang Z, Sadim MS, Sun Z. Stabilized beta-catenin extends thymocyte survival by up-regulating Bcl-xL. J Immunol. 2005;175(12):7981-7988.

44. Gattinoni L, et al. Wnt signaling arrests effector T cell differentiation and generates $\mathrm{CD} 8^{+}$memory stem cells. Nat Med. 2009;15(7):808-813.

45. Sharma A, Chen Q, Nguyen T, Yu Q, Sen JM. T cell factor-1 and $\beta$-catenin control the development of memory-like CD8 thymocytes. J Immunol. 2012;188(8):3859-3868.

46. Wu B, Crampton SP, Hughes CC. Wnt signaling induces matrix metalloproteinase expression and regulates T cell transmigration. Immunity. 2007;26(2):227-239.

47. Xue HH, Zhao DM. Regulation of mature T cell responses by the Wnt signaling pathway. Ann N Y Acad Sci. 2012;1247:16-33.

48. Sancho E, Batlle E, Clevers H. Signaling pathways in intestinal development and cancer. Annu Rev Cell Dev Biol. 2004;20:695-723

49. Crosnier C, Stamataki D, Lewis J. Organizing cell renewal in the intestine: stem cells, signals and combinatorial control. Nat Rev Genet. 2006;7(5):349-359.

50. Beisner J, et al. TCF-1-mediated Wnt signaling regulates Paneth cell innate immune defense effectors HD-5 and -6: implications for Crohn's disease. Am J Physiol Gastrointest Liver Physiol. 2014;307(5):G487-G498.

51. Gulati AS, et al. Mouse background strain profoundly influences Paneth cell function and intestinal microbial composition. PLoS ONE. 2012;7(2):e32403.

52. Round JL, Mazmanian SK. The gut microbiota shapes intestinal immune responses during health and disease. Nat Rev Immunol. 2009;9(5):313-323.

53. Rawls JF, Mahowald MA, Ley RE, Gordon JI. Reciprocal gut microbiota transplants from zebrafish and mice to germ-free recipients reveal host habitat selection. Cell. 2006;127(2):423-433.

54. Round JL, et al. The Toll-like receptor 2 pathway establishes colonization by a commensal of the human microbiota. Science 2011;332(6032):974-977.

55. Longman RS, Yang Y, Diehl GE, Kim SV, Littman DR. Microbiota: host interactions in mucosal homeostasis and systemic autoimmunity. Cold Spring Harb Symp Quant Biol. 2013;78:193-201.

56. Cheroutre H. In IBD eight can come before four. Gastroenterology. 2006;131(2):667-670.

57. Dey N, et al. Regulators of gut motility revealed by a gnotobiotic model of diet-microbiome interactions related to travel. Cell. 2015;163(1):95-107

58. Yoshiya K, et al. Depletion of gut commensal bacteria attenuates intestinal ischemia/reperfusion injury. Am J Physiol Gastrointest Liver Physiol. 2011;301(6):G1020-G1030.

59. Grasa L, et al. Antibiotic-induced depletion of murine microbiota induces mild inflammation and changes in Toll-like receptor patterns and intestinal motility. Microb Ecol. 2015;70(3):835-848.

60. Barbara G, et al. Interactions between commensal bacteria and gut sensorimotor function in health and disease. Am J Gastroenterol. 2005;100(11):2560-2568.

61. Dass NB, et al. The relationship between the effects of short-chain fatty acids on intestinal motility in vitro and GPR43 receptor activation. Neurogastroenterol Motil. 2007;19(1):66-74.

62. Malbert C-H. The ileocolonic sphincter. Neurogastroenterol Motil. 2005;17 Suppl 1:41-49.

63. Osinski MA, Bass P, Gaumnitz EA. Peripheral and central actions of orphanin FQ (nociceptin) on murine colon. Am J Physiol. 1999;276(1 Pt 1):G125-G131.

64. Nagakura Y, Naitoh Y, Kamato T, Yamano M, Miyata K. Compounds possessing 5-HT3 receptor antagonistic activity inhibit intestinal propulsion in mice. Eur J Pharmacol. 1996;311(1):67-72.

65. Sato T, Clevers H. Primary mouse small intestinal epithelial cell cultures. Methods Mol Biol. 2013;945:319-328.

66. Caporaso JG, et al. Ultra-high-throughput microbial community analysis on the Illumina HiSeq and MiSeq platforms. ISME J. 2012;6(8):1621-1624.

67. Magoč T, Salzberg SL. FLASH: fast length adjustment of short reads to improve genome assemblies. Bioinformatics. 2011;27(21):2957-2963.

68. Caporaso JG, et al. QIIME allows analysis of high-throughput community sequencing data. Nat Methods. 2010;7(5):335-336.

69. Haas BJ, et al. Chimeric 16S rRNA sequence formation and detection in Sanger and 454-pyrosequenced PCR amplicons. Genome Res. 2011;21(3):494-504.

70. DeSantis TZ, et al. Greengenes, a chimera-checked 16S rRNA gene database and workbench compatible with ARB. Appl Environ Microbiol. 2006;72(7):5069-5072.

71. Oksanen J, et al. vegan: community ecology package. http://r-forge.r-project.org/. Accessed on February 12, 2014.

72. Faith DP. Phylogenetic pattern and the quantification of organismal biodiversity. Philos Trans R Soc Lond, B, Biol Sci. 1994;345(1311):45-58.

73. Lozupone C, Lladser ME, Knights D, Stombaugh J, Knight R. UniFrac: an effective distance metric for microbial community comparison. ISME J. 2011;5(2):169-172.

74. Wickham H. ggplot2: Elegant Graphics for Data Analysis. New York, New York, USA: Springer-Verlag; 2009

75. Romero R, et al. The vaginal microbiota of pregnant women who subsequently have spontaneous preterm labor and delivery and those with a normal delivery at term. Microbiome. 2014;2:18.

76. Weisburg WG, Barns SM, Pelletier DA, Lane DJ. 16S ribosomal DNA amplification for phylogenetic study. J Bacteriol. 1991;173(2):697-703. 